\title{
Representations of spaces
}

\author{
WOJCIECH CHACHÓLSKI \\ JEROME SCHERER
}

\begin{abstract}
We explain how the notion of homotopy colimits gives rise to that of mapping spaces, even in categories which are not simplicial. We apply the technique of model approximations and use elementary properties of the category of spaces to be able to construct resolutions. We prove that the homotopy category of any monoidal model category is always a central algebra over the homotopy category of Spaces.
\end{abstract}

$55 \mathrm{U} 35,18 \mathrm{G} 55 ; 18 \mathrm{G} 10$

\section{Introduction}

Let $\mathcal{C}$ be a category with a chosen class of weak equivalences $W$ ( $W$ is a collection of morphisms in $\mathcal{C}$ that satisfies the " 2 out of 3 " property and contains all isomorphisms). What does it mean that one can do homotopy theory on $(\mathcal{C}, W)$ ? For us it means three things. First, we should be able to perform basic operations in $\mathcal{C}$ such as pushouts/pull-backs and more generally colimits/limits. Second, the derived versions of these functors (hocolims and holims) should exist. Third, these derived functors should yield a certain action of the category of simplicial sets Spaces on $(\mathcal{C}, W)$ and lead to mapping spaces. Our attitude is that colimits/limits and their derived functors are the fundamental ingredients of homotopy theory and the rest should follow from their properties. This is an alternative to the approach of Hovey presented in [12] and more in the spirit of what we believe we have learned from Bousfield-Kan [1] and Dwyer et al [6].

In this paper we study the following functor:

$$
\otimes_{l}: \text { Spaces } \times \mathcal{C} \rightarrow \operatorname{Ho}(\mathcal{C}) \quad \mathbf{K} \otimes_{l} X:=\operatorname{hocolim}_{\mathbf{K}} c X
$$

where $\operatorname{Ho}(\mathcal{C})=\mathcal{C}\left[W^{-1}\right]$ and $c X: \mathbf{K} \rightarrow \mathcal{C}$ is the constant functor indexed by the simplex category of $K$ (see 2.6) whose value is $X$. We would like $\otimes_{l}$ to be the composition of the localization functor Spaces $\times \mathcal{C} \rightarrow \operatorname{Ho}($ Spaces $) \times \operatorname{Ho}(\mathcal{C})$ and a functor denoted by the same symbol $\otimes_{l}$ : $\operatorname{Ho}($ Spaces $) \times \operatorname{Ho}(\mathcal{C}) \rightarrow \operatorname{Ho}(\mathcal{C})$ (homotopy invariance). The latter should be a homotopy left action (see 2.11 and 2.15), which implies in particular that, for any $X \in \operatorname{Ho}(\mathcal{C})$, the functor $-\otimes_{l} X$ : $\operatorname{Ho}$ (Spaces) $\rightarrow \operatorname{Ho}(\mathcal{C})$ has a right adjoint 
$\operatorname{map}(X,-)$. This is in our view the universal characterization of what mapping spaces should be.

How can this be realized? In order to insure the existence of the localized category $\mathcal{C}\left[W^{-1}\right]$ and the derived functors hocolims and holims satisfying the above conditions, we need to be able to do some homotopical algebra on $(\mathcal{C}, W)$. One way of formalizing this is to assume that $(\mathcal{C}, W)$ can be given a Quillen (simplicial) model structure [16]. Dwyer and Kan explained in [7] how to enrich such a category with an action of Spaces using the so called "hammocks". They also introduce the technique of taking (co)simplicial resolution of the source (target), further developed by Hovey in [12], as well as Hirschhorn in [11] to obtain mapping spaces. Alternatively one could try to find conditions under which a model category is Quillen equivalent to a simplicial model category, as defined by Quillen in [16, Section II.1]. This has been done by Dugger in [5] and Rezk, Shipley, and Schwede in [17]. These approaches however do not seem to emphasize the fundamental role of homotopy colimits and limits. In this context we should mention also the work of Heller [10].

Since in our view hocolims and holims are so important, we need a suitable set up in which such constructions can be effectively studied. It turns out that for that purpose, instead of putting additional assumptions on a model category (cellularity [11], realization axiom [17], etc), it is more advantageous to relax some of the requirements. The aim of this paper is to explain the less restrictive approach of model approximations as introduced in [2]. Recall the following definition.

Definition 1.1 A left model approximation of $(\mathcal{C}, W)$ is a pair of adjoint functors $l: \mathcal{M} \rightleftarrows \mathcal{C}: r$ satisfying the following conditions:

(1) $\mathcal{M}$ is a model category;

(2) $l$ is left adjoint to $r$;

(3) if $f$ is a weak equivalence in $\mathcal{C}$, then $r f$ is a weak equivalence in $\mathcal{M}$;

(4) if $f$ is a weak equivalence between cofibrant objects in $\mathcal{M}$, then $l f$ is a weak equivalence in $\mathcal{C}$;

(5) for any $A \in \mathcal{C}$ and cofibrant $X \in \mathcal{M}$, if $X \rightarrow r A$ is a weak equivalence in $\mathcal{M}$, then its left adjoint $l X \rightarrow A$ is a weak equivalence in $\mathcal{C}$.

A pair of adjoint functors $l: \mathcal{C} \rightleftarrows \mathcal{M}: r$ is a right model approximation of $(\mathcal{C}, W)$ if the duals $r^{\vee}: \mathcal{M}^{\mathrm{op}} \rightleftarrows \mathcal{C}^{\mathrm{op}}: l^{\vee}$ form a left model approximation of $\left(\mathcal{C}^{\mathrm{op}}, W\right)$.

Model approximations have several crucial properties. A category admitting a model approximation (left or right) can be localized with respect to weak equivalences to 
form the homotopy category $\operatorname{Ho}(\mathcal{C})$ [2, Proposition 5.5]. Weak equivalences in such categories are saturated, ie, a morphism in $\mathcal{C}$ belongs to $W$ if and only if the induced morphism in $\operatorname{Ho}(\mathcal{C})$ is an isomorphism. The category $\operatorname{Ho}(\mathcal{C})$ can be identified with a full subcategory of $\operatorname{Ho}(\mathcal{M})$ via the functor $r$ or $l$, depending on whether the approximation is left or right [2, Proposition 5.5]. A left model approximation $l: \mathcal{M} \rightleftarrows \mathcal{C}: r$ leads to a "cofibrant replacement" in $\mathcal{C}$ given by the adjoint $\operatorname{lQr} A \rightarrow A$ of a cofibrant replacement $Q r A \rightarrow r A$ in $\mathcal{M}$ [2, Remark 5.10]. In a dual way a right model approximation leads to a fibrant replacement. These replacements can be used to construct derived functors. If $F: \mathcal{C} \rightarrow \mathcal{D}$ is a functor for which the composition $F l$ sends weak equivalences between cofibrant objects in $\mathcal{M}$ to isomorphisms in $\mathcal{D}$, then the left derived functor of $F$ is given by $A \mapsto F(\operatorname{lQr} A)$ [2, Proposition 5.9, Remark 5.10]. In the same way right model approximations can be used to construct right derived functors. A left model approximation $l: \mathcal{M} \rightleftarrows \mathcal{C}: r$ induces a natural left model approximation on the category of functors $\operatorname{Fun}(I, \mathcal{C})$, for any small category $I$ (see Theorem 6.5). A cofibrant replacement of this model approximation is thus good for constructing the total left derived functors of the colimit functor $\operatorname{colim}_{I}: \operatorname{Fun}(I, \mathcal{C}) \rightarrow \mathcal{C}$. Likewise one constructs the total left derived functor of left Kan extensions, [2, Theorem 11.3(3)]. These derived functors (the homotopy colimits and the homotopy left Kan extensions) are related to each other by various properties. At a 2-categorical level this has been formalized by Cisinski in [3] and amounts to saying that the prederivator associated to any model category is a Grothendieck derivator. The same applies to right model approximations.

To make the approach of model approximations viable we need one more thing. We need to show that a model approximation leads in a natural way to an action of Spaces. The purpose of [2] was to introduce simple geometric techniques to study hocolims and holims. In this paper we are going to illustrate how to use these techniques further, which is what motivated us originally. We did not want to just state and prove some properties of mapping spaces, but more importantly we meant to explain an approach and provide coherent tools to study them. This will be illustrated by proving the following theorem (see Proposition 9.4 and Theorem 10.7).

Theorem Assume that $(\mathcal{C}, W)$ admits a left model approximation. The functor $\otimes_{l}$ : Spaces $\times \mathcal{C} \rightarrow \operatorname{Ho}(\mathcal{C})$ is homotopy invariant and, for any object $X \in \mathcal{C}$, $-\otimes_{l} X: \operatorname{Ho}($ Spaces $) \rightarrow \operatorname{Ho}(\mathcal{C})$ has a right adjoint $\operatorname{map}(X,-)$.

Assume that $(\mathcal{C}, W)$ admits both a left and a right model approximations. Then, for any space $K$, the functor $\mathbf{K} \otimes_{l}-: \operatorname{Ho}(\mathcal{C}) \rightarrow \operatorname{Ho}(\mathcal{C})$ is left adjoint to $X \mapsto \operatorname{holim}_{\mathbf{K}} c X$. 
As an application we prove a conjecture made by Hovey in [12, page 119] about the homotopy category of a monoidal model category. This has been proved independently by Cisinski in his work on Grothendieck derivators, [4].

The plan of the article is as follows. Section 2 is a list of basic definitions and notation. In particular we recall what an action of Spaces on a model category is, and how to construct derived functors. The reader might skip this section and refer to it when needed. In Section 3 we explain that, unlike arbitrary categories, simplex categories behave well with respect to pull-backs. A brief reminder about bounded functors appears then in Section 4. We introduce in Section 5 a simplicial structure on bounded diagrams indexed by a barycentric subdivision. In Section 6 we see how the geometry of the simplex category is reflected in the homotopy theory of bounded diagrams. The next three sections form the core of the paper. Section 7 is basically devoted to prove an invariance property for homotopy colimits of constant diagrams. In Section 8 we show that very little is missing for the simplicial structure to be homotopy invariant; for homotopically constant diagrams, it is so. We are finally ready to construct mapping spaces in Section 9. The theory is dualized in Section 10. In the final Section 11 we illustrate our approach by giving an answer to Hovey's question.

Acknowledgments The first author is supported in part by NSF grant DMS-0296117, Vetenskapsrådet grant 2001-4296, and Göran Gustafssons Stiftelse, the second author by FEDER-MEC grant MTM2004-06686 and the program Ramón y Cajal, MEC, Spain.

\section{Notation and set up}

2.1 Cat denotes the category of small categories and Spaces the category of simplicial sets. A simplicial set is also called a space.

2.2 From Section 3 on, categories denoted by the symbols $\mathcal{C}$ and $\mathcal{D}$ are assumed to contain all colimits and limits, compare with Quillen's axiom (MC0) [16].

2.3 A category with weak equivalences is a category $\mathcal{C}$ with a chosen collection of morphisms $W$, called weak equivalences, which satisfies the " 2 out of 3 " property and contains all isomorphisms. Elements in $W$ are denoted by the symbol $\stackrel{\sim}{\rightarrow}$. The category $\mathcal{C}\left[W^{-1}\right]$, if it exists, is called the homotopy category and is denoted by $\operatorname{Ho}(\mathcal{C})$. By $\pi_{\mathcal{C}}: \mathcal{C} \rightarrow \operatorname{Ho}(\mathcal{C})$ we denote the localization functor.

2.4 The category of all $\mathcal{C}$-valued functors indexed by a small category $I$, with natural transformations as morphisms, is denoted by $\operatorname{Fun}(I, \mathcal{C})$. The symbol $c X: I \rightarrow \mathcal{C}$ denotes 
the constant functor indexed by $I$ with value $X \in \mathcal{C}$. If $(\mathcal{C}, W)$ is a category with weak equivalences, then $\operatorname{Fun}(I, \mathcal{C})$ stands for a category with weak equivalences given by the object-wise weak equivalences. A functor $F: I \rightarrow \mathcal{C}$ is called homotopically constant if there is an object $X \in \mathcal{C}$ and a weak equivalence $F \rightarrow c X$ in $\operatorname{Fun}(I, \mathcal{C})$.

2.5 Let $I$ and $J$ be small categories and $f: I \rightarrow J$ be a functor. The left and right adjoints to $f^{*}: \operatorname{Fun}(J, \mathcal{D}) \rightarrow \operatorname{Fun}(I, \mathcal{D}), F \mapsto F \circ f$, if they exist, are called Kan extensions. They are denoted respectively by $f^{k}: \operatorname{Fun}(I, \mathcal{D}) \rightarrow \operatorname{Fun}(J, \mathcal{D})$ and $f_{k}: \operatorname{Fun}(I, \mathcal{D}) \rightarrow \operatorname{Fun}(J, \mathcal{D})$. These functors can be described explicitly in terms of the over-categories $f \downarrow j$ and under-categories $j \downarrow f$ (Mac Lane [15, II.6]): $\left(f^{k} F\right)(j)=\operatorname{colim}_{f \downarrow j} F$ and $\left(f_{k} F\right)(j)=\lim _{j \downarrow f} F$.

2.6 The simplex category of a simplicial set $K$, denoted by the same letter in a boldface font $\mathbf{K}$, is a category whose objects are simplices of $K$, or equivalently maps of the form $\sigma: \Delta[n] \rightarrow K$. The morphisms in $\mathbf{K}$ between $\sigma: \Delta[n] \rightarrow K$ and $\tau: \Delta[m] \rightarrow K$ are those maps $f: \Delta[n] \rightarrow \Delta[m]$ for which $\sigma=\tau f$. Subject to standard cosimplicial identities, the morphisms in $\mathbf{K}$ are generated by the face and degeneracy morphisms:
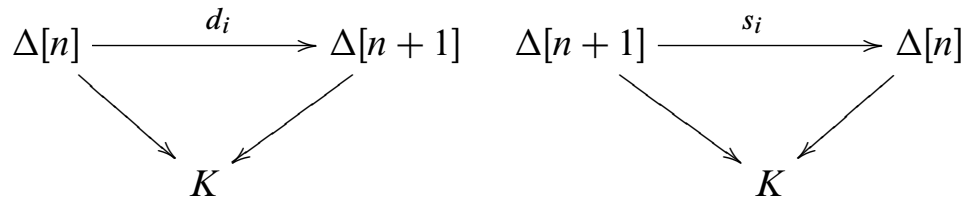

A map of spaces $f: A \rightarrow B$ induces a functor $\mathbf{f}: \mathbf{A} \rightarrow \mathbf{B}$. For any simplex $\sigma \in B$, the functor $\mathbf{f} \downarrow \sigma \rightarrow \mathbf{A}$ is a functor between simplex categories given by the map of spaces $\lim (\Delta[n] \stackrel{\sigma}{\rightarrow} B \stackrel{f}{\leftarrow} A) \rightarrow A$. In particular, when $f$ is the identity, the functor $\mathbf{B} \downarrow \sigma \rightarrow \mathbf{B}$ is induced by the map $\sigma: \Delta[n] \rightarrow B$.

2.7 The functor Spaces $\rightarrow$ Cat, $K \mapsto \mathbf{K}$, has a right adjoint and therefore converts colimits in Spaces into colimits in Cat, see Latch-Thomason-Wilson [14]. This functor also takes pull-backs of spaces into pull-backs of categories. It does not however preserve final objects and consequently neither products: the simplex category of a point is the category of finite ordered sets $\Delta$. For that reason we use the symbol $\mathbf{A} \otimes \mathbf{B}$ to denote the simplex category of $A \times B$. The product of the simplex categories is denoted as usual by $\mathbf{A} \times \mathbf{B}$. The natural inclusion $\mathbf{A} \otimes \mathbf{B} \rightarrow \mathbf{A} \times \mathbf{B}$ is never an equivalence of categories, unless $A$ or $B$ is the empty space.

2.8 $N:$ Cat $\rightarrow$ Spaces denotes the nerve construction [2, Definition 6.3]. It has a left adjoint and thus it converts limits in Cat into limits in Spaces, see [14]. 
2.9 The functor Spaces $\rightarrow$ Spaces, $K \mapsto N(\mathbf{K})$, is called the barycentric subdivision. It commutes with colimits since it is a left adjoint to the functor that associates to a space $L$, the space whose $n$-simplices are $\operatorname{mor}_{\text {Spaces }}(N([n]), L),[2,6.11]$, which is very similar to the so called Kan's Ex construction, [13].

2.10 A functor $f: I \rightarrow J$ between small categories is called a weak equivalence if $N(f)$ is a weak equivalence in Spaces. A map of spaces $f: A \rightarrow B$ is a weak equivalence if and only if the induced functor of simplex categories is a weak equivalence. For any spaces $A$ and $B$, the inclusion $\mathbf{A} \otimes \mathbf{B} \rightarrow \mathbf{A} \times \mathbf{B}$ is a weak equivalence of categories.

2.11 A left action of Spaces on $\mathcal{C}$ is an enrichment of $\mathcal{C}$ over Spaces for which the space of morphisms has a left adjoint in the first variable. A left action of Spaces on $\mathcal{C}$ can be described explicitly as a functor $\otimes$ : Spaces $\times \mathcal{C} \rightarrow \mathcal{C}$ such that:

(1) there are isomorphisms $\Delta[0] \otimes X \cong X$ natural in $X$;

(2) there are coherent isomorphisms $(K \times L) \otimes X \cong K \otimes(L \otimes X)$ natural in $K, L \in$ Spaces and $X \in \mathcal{C}$ (the explicit coherence diagrams can be found in Hovey [12, Definition 4.1.6]);

(3) for any $X \in \mathcal{C},-\otimes X$ : Spaces $\rightarrow \mathcal{C}$ has a right adjoint $\operatorname{map}(X,-)$.

Note that if $\mathcal{C}$ is closed under taking colimits, then condition (3) can be replaced by the requirement:

(3') for any $X \in \mathcal{C},-\otimes X$ : Spaces $\rightarrow \mathcal{C}$ commutes with arbitrary colimits.

Thus what we call in this paper a left action of the category Spaces is a special case of what is called a left Spaces-module structure on $\mathcal{C}$ in [12, Definition 4.1.6], namely one in which the tensor $-\otimes X$ has a right adjoint.

2.12 An enriched functor between categories enriched over Spaces is called simplicial. A simplicial functor between left actions on $\mathcal{C}$ and $\mathcal{D}$ can be described explicitly as a functor $\phi: \mathcal{C} \rightarrow \mathcal{D}$ together with a natural transformation $\mu: K \otimes \phi(X) \rightarrow \phi(K \otimes X)$ which satisfies the following coherence conditions:

(1) the composition $\Delta[0] \otimes \phi(X) \stackrel{\mu}{\rightarrow} \phi(\Delta[0] \otimes X) \cong \phi(X)$ is the natural isomorphism given in 2.11(1); 
(2) for any $X \in \mathcal{C}$ and $K, L \in$ Spaces, the following diagram commutes, where the indicated isomorphisms are induced by the coherent isomorphism in 2.11(2):

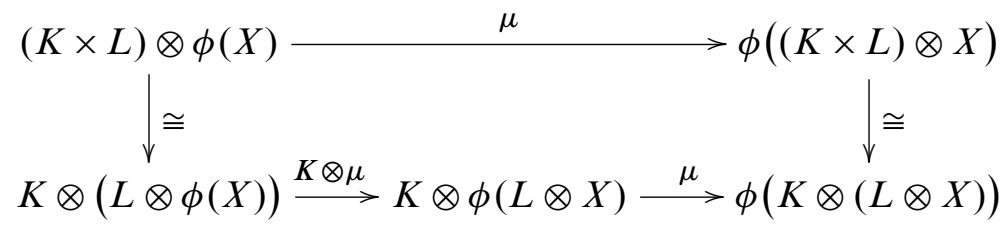

2.13 A right action of Spaces on $\mathcal{C}$ is an enrichment of $\mathcal{C}$ over Spaces, where the space of morphisms has a right adjoint in the second variable. A right action of Spaces on $\mathcal{C}$ can be described explicitly as a functor hom: $\operatorname{Spaces}^{\mathrm{op}} \times \mathcal{C} \rightarrow \mathcal{C}$ such that:

(1d) there are isomorphisms $X \cong \operatorname{hom}(\Delta[0], X)$ natural in $X$;

(2d) there are coherent isomorphism $\operatorname{hom}(K \times L, X) \cong \operatorname{hom}(K, \operatorname{hom}(L, X))$ natural in $X \in \mathcal{C}$ and $K, L \in$ Spaces;

(3d) for any $X \in \mathcal{C}$, hom $(-, X)$ : $\operatorname{Spaces}^{\text {op }} \rightarrow \mathcal{C}$ has a left adjoint $\operatorname{map}(-, X)$.

Note that hom is a right action of Spaces on $\mathcal{C}$ if and only if hom ${ }^{\mathrm{op}}$ is a left action of Spaces on $\mathcal{C}^{\text {op }}$.

A simplicial functor between right actions of Spaces on $\mathcal{C}$ and $\mathcal{D}$ can be described in a explicit way as a functor $\phi: \mathcal{C} \rightarrow \mathcal{D}$ together with a natural transformation $\phi\left(\operatorname{hom}_{\mathcal{C}}(K, Y)\right) \rightarrow \operatorname{hom}_{\mathcal{D}}(K, \phi(X))$ satisfying coherence conditions analogous to those in 2.12 .

2.14 A simplicial structure on $\mathcal{C}$ is an enrichment of $\mathcal{C}$ over Spaces where the space of morphisms has a left adjoint in the first variable and a right adjoint in the second variable. If such an enrichment has been fixed, $\mathcal{C}$ is called a simplicial category.

A simplicial structure can be described explicitly in two dual ways; first, as a left action $\otimes:$ Spaces $\times \mathcal{C} \rightarrow \mathcal{C}$ which satisfies the extra condition:

(4) The functor $K \otimes-: \mathcal{C} \rightarrow \mathcal{C}$ has a right adjoint $\operatorname{hom}(K,-)$ for any $K$.

In this case the assignment $K \mapsto \operatorname{hom}(K, X)$ can be extended to a functor Spaces ${ }^{\text {op }} \rightarrow \mathcal{C}$ which is the right adjoint to $\operatorname{map}(-, X)$. In particular hom defines a right action of Spaces on $\mathcal{C}$.

Dually a simplicial structure on $\mathcal{C}$ can also be described as a right action

$$
\operatorname{hom}(-,-): \text { Spaces }^{\text {op }} \times \mathcal{C} \rightarrow \mathcal{C}
$$

for which: 
(4d) $\operatorname{hom}(K,-): \mathcal{C} \rightarrow \mathcal{C}$ has a left adjoint $K \otimes-$, for any space $K$.

2.15 If instead of Spaces we take the homotopy category Ho(Spaces) in the above explicit descriptions of actions, then we get the notions of homotopy (left/right) actions of Spaces on $\mathcal{C}$ and simplicial functors. A weak simplicial structure on $\mathcal{C}$ is, by definition, a homotopy action. If such an action is fixed, $\mathcal{C}$ is called a weak simplicial category.

2.16 If $\otimes: \operatorname{Ho}$ (Spaces) $\times \mathcal{C} \rightarrow \mathcal{C}$ is a homotopy left action, then the set of morphisms $\operatorname{mor}_{\mathcal{C}}(X, Y)$ can be identified with the set of connected components $\pi_{0} \operatorname{map}(X, Y)$. It follows that a simplicial functor between homotopy left actions on $\mathcal{C}$ and $\mathcal{D}$ induces a natural transformation $\operatorname{map}_{\mathcal{C}}(X, Y) \rightarrow \operatorname{map}_{\mathcal{D}}(\phi(X), \phi(Y))$ which on the set of connected components coincides with $\phi$. The same applies to simplicial functors of homotopy right actions.

2.17 Let $(\mathcal{C}, W)$ and $(\mathcal{D}, V)$ be categories with weak equivalences whose homotopy categories exist. The total left derived functor of $\Phi: \mathcal{C} \rightarrow \mathcal{D}$ is the left Kan extension [15] of $\pi_{\mathcal{C}}: \mathcal{C} \rightarrow \operatorname{Ho}(\mathcal{C})$ applied to the composition of $\Phi$ and $\pi_{\mathcal{D}}: \mathcal{D} \rightarrow \operatorname{Ho}(\mathcal{D})$. Explicitly, it is a functor $L \Phi: \mathcal{C} \rightarrow \operatorname{Ho}(\mathcal{D})$ with a natural transformation $L \Phi \rightarrow \pi_{D} \Phi$ which is terminal among natural transformations with the range $\pi_{D} \Phi$ and whose domains are functors factoring through $\pi_{\mathcal{C}}: \mathcal{C} \rightarrow \operatorname{Ho}(\mathcal{C})$.

A left replacement for $\Phi: \mathcal{C} \rightarrow \mathcal{D}$ is a functor $Q: \mathcal{C} \rightarrow \mathcal{C}$ and a natural morphism $Q X \rightarrow X$ in $\mathcal{C}$ which is a weak equivalence such that $\pi_{D} \Phi Q \rightarrow \pi_{D} \Phi$ is the total left derived functor of $\Phi$.

The total right derived functor of $\Phi: \mathcal{C} \rightarrow \mathcal{D}$ is the right Kan extension [15] of $\pi_{\mathcal{C}}: \mathcal{C} \rightarrow$ $\operatorname{Ho}(\mathcal{C})$ applied to the composition of $\pi_{\mathcal{D}}: \mathcal{D} \rightarrow \operatorname{Ho}(\mathcal{D})$ and $\Phi$. A right replacement for $\Phi$ is a functor $R: \mathcal{C} \rightarrow \mathcal{C}$ and a natural morphism $X \rightarrow R X$ in $\mathcal{C}$ which is a weak equivalence such that $\pi_{D} \Phi \rightarrow \pi_{D} \Phi R$ is the total right derived functor of $\Phi$.

2.18 We say that $(\mathcal{C}, W)$ can be left localized if, for any small category $I$, the homotopy category of $\operatorname{Fun}(I, \mathcal{C})$ exists and the functor $\operatorname{colim}_{I}: \operatorname{Fun}(I, \mathcal{C}) \rightarrow \mathcal{C}$ has a left replacement. In particular the total left derived functor of $\operatorname{colim}_{I}$ exists and is denoted by $\operatorname{hocolim}_{I}: \operatorname{Fun}(I, \mathcal{C}) \rightarrow \operatorname{Ho}(\mathcal{C})$. It is given by the formula $\operatorname{hocolim}_{I} F=$ $\pi_{\mathcal{C}}\left(\operatorname{colim}_{I} Q F\right)$.

We say that $(\mathcal{C}, W)$ can be right localized if, for any small category $I$, the homotopy category of $\operatorname{Fun}(I, \mathcal{C})$ exists and the functor $\lim _{I}: \operatorname{Fun}(I, \mathcal{C}) \rightarrow \mathcal{C}$ has a right replacement. In particular the total right derived functor of $\lim _{I}$ exists and is denoted by $\operatorname{holim}_{I}: \operatorname{Fun}(I, \mathcal{C}) \rightarrow \operatorname{Ho}(\mathcal{C})$. 
2.19 Assume that $(\mathcal{C}, W)$ can be left localized. By the universal property, for any $F: J \rightarrow \mathcal{C}$ and $f: I \rightarrow J$, there is a morphism $\operatorname{hocolim}_{I} f^{*} F \rightarrow \operatorname{hocolim}_{J} F$ in $\operatorname{Ho}(\mathcal{C})$ natural with respect to $f$ and $F$. This morphism can be described in terms of left replacements $Q_{I}$ and $Q_{J}$ of $\operatorname{colim}_{I}$ and $\operatorname{colim}_{J}$. It is represented by the following sequence of morphisms in $\mathcal{C}$ :

$$
\operatorname{colim}_{I}\left(Q_{I} f^{*} F\right) \widetilde{\leftarrow} \operatorname{colim}_{I}\left(Q_{I} f^{*} Q_{J} F\right) \rightarrow \operatorname{colim}_{I}\left(f^{*} Q_{J} F\right) \rightarrow \operatorname{colim}_{J}\left(Q_{J} F\right) .
$$

In the case $J$ is the category with one object and one morphism, this leads to a functor:

$$
\otimes_{l}: \text { Cat } \times \mathcal{C} \rightarrow \operatorname{Ho}(\mathcal{C}) \quad I \otimes_{l} X:=\operatorname{hocolim}_{I} c X .
$$

Dually, when $(\mathcal{C}, W)$ can be right localized, by the universal property, for any $F: J \rightarrow \mathcal{C}$ and $f: I \rightarrow J$, there is a morphism $\operatorname{holim}_{J} F \rightarrow \operatorname{holim}_{I} f^{*} F$ in $\operatorname{Ho}(\mathcal{C})$ natural with respect to $f$ and $F$. In the case $J$ is the category with one object and one morphism, this leads to a functor:

$$
\text { rhom: } \mathrm{Cat}^{\mathrm{op}} \times \mathcal{C} \rightarrow \operatorname{Ho}(\mathcal{C}) \operatorname{rhom}(I, X):=\operatorname{holim}_{I} c X .
$$

2.20 Assume that $(\mathcal{C}, W)$ and $(\mathcal{D}, V)$ are categories that can be left localized. Let $I$ be a small category, $F: I \rightarrow \mathcal{C}$ be a functor, and $Q_{\mathcal{C}}$ and $Q_{\mathcal{D}}$ be left replacements of $\operatorname{colim}_{I}: \operatorname{Fun}(I, \mathcal{C}) \rightarrow \mathcal{C}$ and $\operatorname{colim}_{I}: \operatorname{Fun}(I, \mathcal{D}) \rightarrow \mathcal{D}$ respectively. If $\Phi: \mathcal{C} \rightarrow \mathcal{D}$ is a functor that sends weak equivalences in $\mathcal{C}$ to weak equivalences in $\mathcal{D}$, then the following sequence of morphisms in $\mathcal{D}$ :

$$
\operatorname{colim}_{I} Q_{\mathcal{D}} \Phi F \leftarrow \operatorname{colim}_{I} Q_{\mathcal{D}} \Phi Q_{\mathcal{C}} F \rightarrow \operatorname{colim}_{I} \Phi Q_{\mathcal{C}} F \rightarrow \Phi\left(\operatorname{colim}_{I} Q_{\mathcal{C}} F\right)
$$

leads to a morphism hocolim $I \Phi F \rightarrow \Phi\left(\operatorname{hocolim}_{I} F\right)$ in $\operatorname{Ho}(\mathcal{D})$. This morphism is natural in $F$ and does not depend on the choice of the left replacements $Q_{\mathcal{C}}$ and $Q_{\mathcal{D}}$.

Dually, let $(\mathcal{C}, W)$ and $(\mathcal{D}, V)$ be categories that can be right localized. Assume that $\Phi: \mathcal{C} \rightarrow \mathcal{D}$ sends weak equivalences in $\mathcal{C}$ to weak equivalences in $\mathcal{D}$. As in the case of hocolims, for any small category $I$ and any functor $F: I \rightarrow \mathcal{C}$, there is a natural morphism $\Phi\left(\operatorname{holim}_{I} F\right) \rightarrow \operatorname{holim}_{I} \Phi F$ in $\operatorname{Ho}(\mathcal{D})$.

\section{Simplex categories}

To understand actions of Spaces one can try to look at the problem locally and study functors indexed by simplex categories 2.6. Such functors are called representations of the indexing space. What is so special about simplex categories that enables us to say something about their representations? 
Simplex categories have one important advantage over arbitrary small categories of which we give now an elementary illustration. Let $g: I \rightarrow X$ be a functor of small categories and $G: I \rightarrow \mathcal{C}$ be a functor. Let us look for conditions under which the operation $G \mapsto g^{k} G$ commutes with the base change. Explicitly, consider a pull-back of small categories:

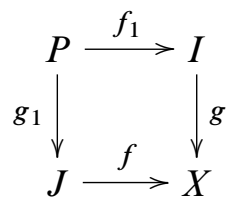

For any $j \in J$, there is a functor of over-categories $g_{1} \downarrow j \rightarrow g \downarrow f(j)$. These functors induce a natural transformation:

$$
\left(g_{1}^{k} f_{1}^{*} G\right)(j)=\operatorname{colim}_{g_{1} \downarrow j} f_{1}^{*} G \rightarrow \operatorname{colim}_{g \downarrow f(j)} G=\left(f^{*} g^{k} G\right)(j) .
$$

We ask: when is this natural transformation an isomorphism? If $G=g^{*} F$, for some $F: X \rightarrow \mathcal{C}$, this natural transformation is of the form:

$$
g_{1}{ }^{k} g_{1}{ }^{*} f^{*} F=g_{1}{ }^{k} f_{1}^{*} g^{*} F \rightarrow f^{*} g^{k} g^{*} F .
$$

We could then apply $f^{k}$ to it and ask: when is $\left(f g_{1}\right)^{k}\left(f g_{1}\right)^{*} F \rightarrow f^{k} f^{*} g^{k} g^{*} F$ an isomorphism? In particular, when is the operation $F \mapsto f^{k} f^{*} g^{k} g^{*} F$ symmetric with respect to $f$ and $g$ ?

Let us look at two examples.

Example 3.1 First a negative case. Consider a pull-back of categories:

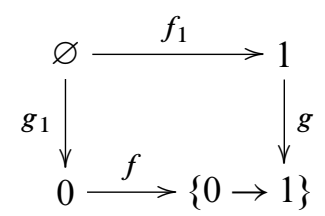

and a functor $\{A \rightarrow B\}:\{0 \rightarrow 1\} \rightarrow$ Spaces. It can be verified that:

$$
g^{k} g^{*}\{A \rightarrow B\}=\{\varnothing \rightarrow B\} \text { and } f^{k} f^{*}\{A \rightarrow B\}=\{A \stackrel{\text { id }}{\rightarrow} A\} \text {. }
$$

In particular $f^{k} f^{*} g^{k} g^{*}\{A \rightarrow B\}=\{\varnothing \rightarrow \varnothing\}$ does not coincide with the functor $g^{k} g^{*} f^{k} f^{*}\{A \rightarrow B\}=\{\varnothing \rightarrow A\}$ in general. 
Here is a positive case. Consider a pull-back of categories:

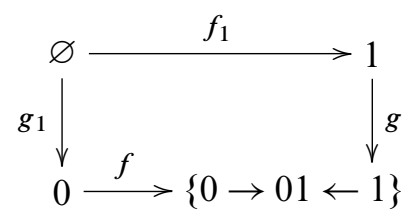

and a functor $\{A \rightarrow B \leftarrow C\}:\{0 \rightarrow 01 \leftarrow 1\} \rightarrow$ Spaces. In this case:

$$
\begin{aligned}
& g^{k} g^{*}\{A \rightarrow B \leftarrow C\}=\{\varnothing \rightarrow C \stackrel{\text { id }}{\leftarrow} C\} \\
& f^{k} f^{*}\{A \rightarrow B \leftarrow C\}=\{A \stackrel{\text { id }}{\rightarrow} A \leftarrow \varnothing\} .
\end{aligned}
$$

In particular:

$$
\begin{aligned}
& f^{k} f^{*} g^{k} g^{*}\{A \rightarrow B \leftarrow C\}=\{\varnothing \rightarrow \varnothing \leftarrow \varnothing\} \\
& g^{k} g^{*} f^{k} f^{*}\{A \rightarrow B \leftarrow C\}=\{\varnothing \rightarrow \varnothing \leftarrow \varnothing\}
\end{aligned}
$$

which agrees with $\left(g f_{1}\right)^{k}\left(g f_{1}\right)^{*}\{A \rightarrow B \leftarrow C\}$.

What is so special about the second example? It turns out that it is an example of functors indexed by simplex categories. The reason this does not work in general is the fact that arbitrary categories can have vastly different over-categories. Simplex categories on the other hand look the same locally. For any simplex $\sigma: \Delta[n] \rightarrow K$, the functor from the over-category $\mathbf{K} \downarrow \sigma \rightarrow \mathbf{K}$ is isomorphic to the functor induced by the map $\sigma: \Delta[n] \rightarrow K$. Thus, locally, all simplex categories look like standard simplices.

Proposition 3.2 Let the following be a pull-back square of spaces:

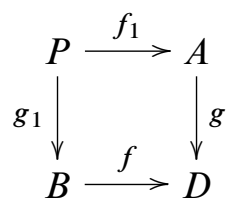

For any functor $G: \mathbf{A} \rightarrow \mathcal{C}$, the natural transformation $\mathbf{g}_{1}{ }^{k} \mathbf{f}_{1}{ }^{*} G \rightarrow \mathbf{f}^{*} \mathbf{g}^{k} G$ is an isomorphism. In particular, for any figure $F: \mathbf{D} \rightarrow \mathcal{C}$, the natural transformations $\mathbf{g}_{1}{ }^{k} \mathbf{g}_{1}{ }^{*} \mathbf{f}^{*} F \rightarrow \mathbf{f}^{*} \mathbf{g}^{k} \mathbf{g}^{*} F$ and $\left(\mathbf{f g}_{1}\right)^{k}\left(\mathbf{f} \mathbf{g}_{1}\right)^{*} F \rightarrow \mathbf{f}^{k} \mathbf{f}^{*} \mathbf{g}^{k} \mathbf{g}^{*} F$ are isomorphisms and the operation $F \mapsto \mathbf{f}^{k} \mathbf{f}^{*} \mathbf{g}^{k} \mathbf{g}^{*} F$ is symmetric with respect to $f$ and $g$.

Proof Note that for any simplex $\sigma: \Delta[n] \rightarrow B$, the over-categories $\mathbf{g}_{1} \downarrow \sigma$ and $\mathbf{g} \downarrow f(\sigma)$ can be identified with the simplex category of the pull-back:

$$
\lim \left(\Delta[n] \stackrel{\sigma}{\rightarrow} B \stackrel{g_{1}}{\leftarrow} P\right) .
$$

Moreover the functor $\mathbf{g}_{1} \downarrow \sigma \rightarrow \mathbf{g} \downarrow f(\sigma)$ is an isomorphism. 


\section{Bounded functors}

Simplex categories have another advantage. There are a lot of geometric constructions that can be performed on spaces. These constructions often translate well to operations on representations. For example, fix a functor $H: I \rightarrow$ Spaces. The simplex category of $\operatorname{colim}_{I} H$ can be identified with the colimit of $\mathbf{H}$ in the category of small categories. A covariant functor $F$ indexed by $\operatorname{colim}_{I} \mathbf{H}$ consists of the following data:

(1) for every object $i$ in $I$, a functor $F_{i}: \mathbf{H}(i) \rightarrow \mathcal{C}$;

(2) for every morphism $\alpha: i \rightarrow j$ in $I, F_{i}: \mathbf{H}(i) \rightarrow \mathcal{C}$ should coincide with the composition $\mathbf{H}(i) \stackrel{\mathbf{H}(\alpha)}{\longrightarrow} \mathbf{H}(j) \stackrel{F_{j}}{\longrightarrow} \mathcal{C}$.

This decomposition yields an isomorphism $\operatorname{colim}_{\operatorname{colim}_{I} \mathbf{H}} F \cong \operatorname{colim}_{I} \operatorname{colim}_{\mathbf{H}(i)} F_{i}$, see [2, Proposition 8.2].

By applying the above procedure inductively, we can calculate colimits using the cell decomposition of the indexing space. Diagrams indexed by the standard simplices $\Delta[n]$ play the role of fundamental building blocks in this process. Unfortunately representations of $\Delta[n]$ are complicated. For example, the simplex category of $\Delta[0]$ is equivalent to the category of finite ordered sets and so its representations are cosimplicial and simplicial objects, depending on variance. For that reason we need to make additional assumptions on the functors considered. We restrict our attention to diagrams that reflect the geometry of the indexing space more directly. A functor $F$, indexed by a simplex category $\mathbf{A}$, is called bounded [2, Definition 10.1] if it assigns an isomorphism to any morphism of the form $s_{i}: \Delta[n+1] \rightarrow \Delta[n]$ in $\mathbf{A}$.

If $S$ denotes the set of all these degeneracy morphisms in $\mathbf{A}$, then a bounded functor is nothing else but a functor indexed by the localized category $\mathbf{A}\left[S^{-1}\right]$. Depending on the variance, the categories of bounded diagrams are denoted by $\operatorname{Fun}^{b}(\mathbf{A}, \mathcal{C})$ and $\operatorname{Fun}^{b}\left(\mathbf{A}^{\mathrm{op}}, \mathcal{C}\right)$.

An important observation is that the degeneracy morphisms in A do not form any essential loop. Any bounded functor is naturally isomorphic to a functor that assigns identities to morphisms in $S$ [2, Proposition 10.3]. This can be therefore assumed about any considered bounded functor.

Example 4.1 The "triviality" of $S$ does not imply in general that the localization functor $\mathbf{A} \rightarrow \mathbf{A}\left[S^{-1}\right]$ is a weak equivalence (see 2.10). Let:

$$
A=\operatorname{colim}(\Delta[3] / \Delta[2] \stackrel{\pi}{\leftarrow} \Delta[3,1] \hookrightarrow \Delta[3])
$$

Algebraic 83 Geometric Topology, Volume 8 (2008) 
where $\pi: \Delta[3,1] \rightarrow \Delta[3] / \Delta[2]$ is the composition of $\Delta[3,1] \hookrightarrow \Delta[3]$ and the quotient map $d_{1}: \Delta[2] \rightarrow \Delta[3] \rightarrow \Delta[3] / \Delta[2]$. Although $A$ is contractible, the category $\mathbf{A}\left[S^{-1}\right]$ is not. The nerve $N\left(\mathbf{A}\left[S^{-1}\right]\right)$ is weakly equivalent to $S^{2}$.

Let $A=\Delta[n] / \partial \Delta[n]$. For $n>1$, the category $\mathbf{A}\left[S^{-1}\right]$ is equivalent to $* \rightarrow *$ (the category with two objects and one non-identity morphism).

It is clear that, for any map of spaces $f: A \rightarrow B, \mathbf{f}^{*}: \operatorname{Fun}(\mathbf{B}, \mathcal{C}) \rightarrow \operatorname{Fun}(\mathbf{A}, \mathcal{C})$ takes bounded functors to bounded functors. The same is also true for the left Kan extension $\mathbf{f}^{k}: \operatorname{Fun}(\mathbf{A}, \mathcal{C}) \rightarrow \operatorname{Fun}(\mathbf{B}, \mathcal{C})$ [2, Theorem 33.1]. It follows that the functors $\mathbf{f}^{k}: \operatorname{Fun}^{b}(\mathbf{A}, \mathcal{C}) \rightleftarrows \operatorname{Fun}^{b}(\mathbf{B}, \mathcal{C}): \mathbf{f}^{*}$ form an adjoint pair.

\section{Subdivision}

In this section we study bounded functors indexed by barycentric subdivisions. Recall that the subdivision of $A$ is given by the nerve $N(\mathbf{A})$ of the simplex category of a space $A$ (see 2.9).

A map of spaces $f: A \rightarrow B$ is called reduced if it sends non-degenerate simplices in $A$ to non-degenerate simplices in $B$ [2, Definition 12.9]. Reduced maps can be characterized using a lifting test: the map $f: A \rightarrow B$ is reduced if and only if in any commutative diagram of the form:

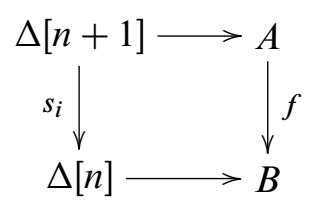

there is a lift, ie a morphism $\Delta[n] \rightarrow A$ for which the resulting diagram with five arrows commutes [2, Proposition 18.5]. It follows that a pull-back of a reduced map is also reduced.

The subdivision operation has two crucial properties. The first is the following proposition.

Proposition 5.1 [2, Example 12.10] For any map of spaces $f: A \rightarrow B$, the induced map $N(\mathbf{f}): N(\mathbf{A}) \rightarrow N(\mathbf{B})$ is reduced.

Second, the subdivision $N(\mathbf{A})$ contains enough combinatorial information to induce a non-trivial simplicial structure on $\operatorname{Fun}^{b}(\mathbf{N}(\mathbf{A}), \mathcal{C})$ (the non-triviality will follow from Corollary 8.6). Here is the description of this structure. Choose a space $K$ and denote 
by $\pi: N(\mathbf{K} \otimes \mathbf{A}) \rightarrow N(\mathbf{A})$ the subdivision of the projection $p_{K}: K \times A \rightarrow A$ onto the second factor. Define:

$$
K \otimes F:=\pi^{k} \pi^{*} F .
$$

This construction is natural in $F$ and $K$ and hence defines a functor:

$$
\otimes: \text { Spaces } \times \operatorname{Fun}^{b}(\mathbf{N}(\mathbf{A}), \mathcal{C}) \rightarrow \operatorname{Fun}^{b}(\mathbf{N}(\mathbf{A}), \mathcal{C}) .
$$

Example 5.2 In general, we do not know any explicit formula describing $K \otimes F$ in terms of the values of $F$. However if $F$ is a composition of $G: \mathbf{A}^{\text {op }} \rightarrow \mathcal{C}$ and $\epsilon: \mathbf{N}(\mathbf{A}) \rightarrow \mathbf{A}^{\mathrm{op}}, \epsilon\left(\sigma_{n} \rightarrow \sigma_{n-1} \rightarrow \cdots \rightarrow \sigma_{0}\right):=\sigma_{n}$, then $K \otimes F$ is given by:

$$
\left(\sigma_{n} \rightarrow \sigma_{n-1} \rightarrow \cdots \rightarrow \sigma_{0}\right) \mapsto \coprod_{K_{\left|\sigma_{n}\right|}} G\left(\sigma_{n}\right) .
$$

This formula should be compared with the simplicial structure introduced by Quillen on simplicial objects in [16, II.1 Proposition 2].

Proposition 5.3 For any space $A$, the functor $\otimes$ defines a simplicial structure on $\operatorname{Fun}^{b}(\mathbf{N}(\mathbf{A}), \mathcal{C})$ (see 2.14 and 2.11).

Proof We need to check four conditions (see 2.14). It is clear that the natural morphism $\Delta[0] \otimes F=\mathrm{id}^{k} \mathrm{id}^{*} F \rightarrow F$ is an isomorphism and so the first requirement is satisfied. Consider two spaces $K$ and $L$ and a pull-back square:

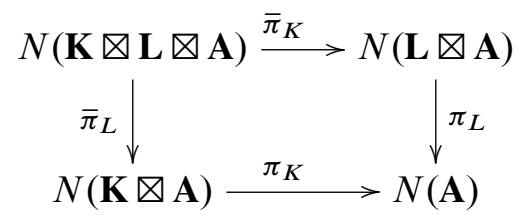

According to Proposition 3.2, for any $F: \mathbf{N}(\mathbf{A}) \rightarrow \mathcal{C}$, the transformation:

$(K \times L) \otimes F=\left(\pi_{K} \bar{\pi}_{L}\right)^{k}\left(\pi_{K} \bar{\pi}_{L}\right)^{*} F \rightarrow\left(\pi_{K}\right)^{k}\left(\pi_{K}\right)^{*}\left(\pi_{L}\right)^{k}\left(\pi_{L}\right)^{*} F=K \otimes(L \otimes F)$ is an isomorphism. Coherence of these isomorphisms follows directly from the coherence of products in Spaces, which proves the second requirement.

To prove condition (3'), we need to show that, for a fixed $F \in \operatorname{Fun}^{b}(\mathbf{N}(\mathbf{A}), \mathcal{C})$, the functor $-\otimes F$ commutes with arbitrary colimits. Let $H: I \rightarrow$ Spaces be a functor. Observe that the map $\operatorname{colim}_{I}(H \times A) \rightarrow\left(\operatorname{colim}_{I} H\right) \times A$ is an isomorphism of spaces and so is the map $\operatorname{colim}_{I} N(\mathbf{H} \otimes \mathbf{A}) \rightarrow N\left(\left(\operatorname{colim}_{I} \mathbf{H}\right) \otimes \mathbf{A}\right)$ as the subdivision commutes with arbitrary colimits (see 2.9). Next, choose a simplex $\sigma: \Delta[n] \rightarrow N(\mathbf{A})$ and define:

$$
P(i):=\lim \left(\Delta[n] \stackrel{\sigma}{\rightarrow} N(\mathbf{A}) \stackrel{\pi_{i}}{\leftarrow} N(\mathbf{H}(i) \otimes \mathbf{A})\right)
$$


where $\pi_{i}$ is the subdivision of the projection $\mathrm{pr}_{2}: H(i) \times A \rightarrow A$. The colimit of $P$ can be identified with the pull-back:

$$
\operatorname{colim}_{I} P \cong \lim \left(\Delta[n] \stackrel{\sigma}{\rightarrow} N(\mathbf{A}) \stackrel{\pi}{\leftarrow} N\left(\left(\operatorname{colim}_{I} \mathbf{H}\right) \otimes \mathbf{A}\right)\right) .
$$

These isomorphisms yield the following identifications:

$$
\begin{aligned}
\left(\left(\operatorname{colim}_{I} H\right) \otimes F\right)(\sigma) & =\left(\pi^{k} \pi^{*} F\right)(\sigma) \cong \operatorname{colim}_{\operatorname{colim}_{I} \mathbf{P}} F \cong \operatorname{colim}_{i \in I} \operatorname{colim}_{\mathbf{P}(i)} F \\
& =\operatorname{colim}_{i \in I}\left(\left(\pi_{i}\right)^{k}\left(\pi_{i}\right)^{*} F\right)(\sigma)=\operatorname{colim}_{I}(H \otimes F)(\sigma) .
\end{aligned}
$$

Note that for a fixed $K$, the functor $K \otimes-$ has a right adjoint as it is a composition of two functors that admit right adjoints (here we use our general assumption that $\mathcal{C}$ has all limits, see 2.2). This proves the fourth requirement.

Taking the subdivision in Proposition 5.3 is crucial. In a similar way one could define a simplicial structure on $\operatorname{Fun}^{b}(\mathbf{A}, \mathcal{C})$ by $K \otimes F:=\left(\mathbf{p}_{K}\right)^{k}\left(\mathbf{p}_{K}\right)^{*} F$, where $p_{K}: K \times A \rightarrow A$ is the projection onto the second factor. This however does not give anything interesting as the obtained structure is trivial.

5.4 Naturality How functorial is this simplicial structure on $\operatorname{Fun}^{b}(\mathbf{N}(A), \mathcal{C})$ ? Consider the following pull-back of spaces associated with a map $f: A \rightarrow B$ and a space $K$ :

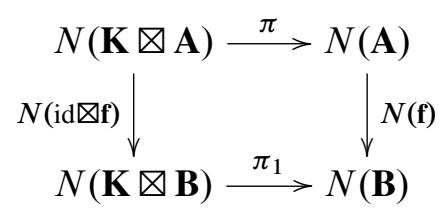

where the horizontal maps are the subdivisions of the projections. For any bounded diagram $F \in \operatorname{Fun}^{b}(\mathbf{N}(\mathbf{B}), \mathcal{C})$, there is a natural transformation:

$$
K \otimes\left(\mathbf{N}(\mathbf{f})^{*} F\right)=\pi^{k} \pi^{*} \mathbf{N}(\mathbf{f})^{*} F \rightarrow \mathbf{N}(\mathbf{f})^{*}\left(\pi_{1}\right)^{k}\left(\pi_{1}\right)^{*} F=\mathbf{N}(\mathbf{f})^{*}(K \otimes F)
$$

which is an isomorphism by Proposition 3.2. Note that this isomorphism is natural with respect to the variables $K$ and $F$. Together with this isomorphism the functor $\mathbf{N}(\mathbf{f})^{*}: \operatorname{Fun}^{b}(\mathbf{N}(\mathbf{B}), \mathcal{C}) \rightarrow \operatorname{Fun}^{b}(\mathbf{N}(\mathbf{A}), \mathcal{C})$ becomes a simplicial functor (see 2.12).

For any $G \in \operatorname{Fun}^{b}(\mathbf{N}(\mathbf{A}), \mathcal{C})$, there is a natural transformation:

$$
\mathbf{N}(\mathrm{id} \otimes \mathbf{f})^{k} \pi^{*} G \rightarrow\left(\pi_{1}\right)^{*} \mathbf{N}(\mathbf{f})^{k} G
$$

which is an isomorphism again by Proposition 3.2. We can apply $\left(\pi_{1}\right)^{k}$ to it and get a natural isomorphism:

$$
\mathbf{N}(\mathbf{f})^{k}(K \otimes G)=\left(\pi_{1}\right)^{k} \mathbf{N}(\mathrm{id} \otimes \mathbf{f})^{k} \pi^{*} G \rightarrow\left(\pi_{1}\right)^{k}\left(\pi_{1}\right)^{*} \mathbf{N}(\mathbf{f})^{k} G=K \otimes\left(\mathbf{N}(\mathbf{f})^{k} G\right)
$$


where we identified $K \otimes G=\pi^{k} \pi^{*} G$ and used the commutativity of the above pullback square. It is not difficult to see that this isomorphism is natural with respect to $K$ and $G$. The functor $\mathbf{N}(\mathbf{f})^{k}: \operatorname{Fun}^{b}(\mathbf{N}(\mathbf{A}), \mathcal{C}) \rightarrow \operatorname{Fun}^{b}(\mathbf{N}(\mathbf{B}), \mathcal{C})$, together with the inverse of this isomorphism, becomes a simplicial functor.

Let $\phi: \mathcal{C} \rightarrow \mathcal{D}$ be a functor and $K$ be a simplicial set. Consider the following pull-back of spaces:

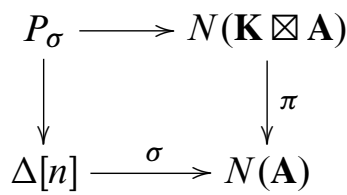

By the universal property of the colimit construction, for any $F: \mathbf{N}(\mathbf{A}) \rightarrow \mathcal{C}$, there is a morphism in $\mathcal{D}$ :

$$
(K \otimes(\phi F))(\sigma)=\operatorname{colim}_{\mathbf{P}_{\sigma}}(\phi F \pi) \rightarrow \phi\left(\operatorname{colim}_{\mathbf{P}_{\sigma}} F \pi\right)=\phi(K \otimes F)(\sigma) .
$$

These morphisms form a natural transformation $K \otimes(\phi F) \rightarrow \phi(K \otimes F)$ functorial with respect to the variables $K$ and $F$. With this natural transformation, the functor $\phi_{*}: \operatorname{Fun}^{b}(\mathbf{N}(\mathbf{A}), \mathcal{C}) \rightarrow \operatorname{Fun}^{b}(\mathbf{N}(\mathbf{A}), \mathcal{D})$ becomes a simplicial functor.

\section{Homotopy theory of bounded functors}

Simplex categories are also rather special from the homotopy point of view.

Theorem 6.1 [2, Theorem 21.1] Let $\mathcal{M}$ be a model category. The following describes a model structure on $\operatorname{Fun}^{b}(\mathbf{A}, \mathcal{M})$ :

- $\phi: F \rightarrow G$ is a weak equivalence (fibration) if, for any simplex $\sigma \in A$, $\phi_{\sigma}: F(\sigma) \rightarrow G(\sigma)$ is a weak equivalence (fibration) in $\mathcal{M}$;

- $\phi: F \rightarrow G$ is an (acyclic) cofibration if, for any non-degenerate simplex $\sigma: \Delta[n] \rightarrow A$, the morphism:

$$
\operatorname{colim}\left(F(\sigma) \leftarrow \operatorname{colim}_{\partial \Delta[n]} F \rightarrow \operatorname{colim}_{\partial \Delta[n]} G\right) \rightarrow G(\sigma)
$$

is an (acyclic) cofibration in $\mathcal{M}$.

The description of (acyclic) cofibrations in Theorem 6.1 can be used by induction on the cell attachment to show the following proposition. 
Proposition 6.2 [2, Proposition 20.1] If $\phi: F \rightarrow G$ is an (acyclic) cofibration in Fun $^{b}(\mathbf{A}, \mathcal{M})$, then, for any subspace $X \subset A$, the morphism:

$$
\operatorname{colim}\left(\operatorname{colim}_{\mathbf{X}} G \stackrel{\phi}{\leftarrow} \operatorname{colim}_{\mathbf{X}} F \rightarrow \operatorname{colim}_{\mathbf{A}} F\right) \hookrightarrow \operatorname{colim}_{\mathbf{A}} G
$$

is an (acyclic) cofibration in $\mathcal{M}$.

How functorial is this model structure?

Proposition 6.3 Let $f: A \rightarrow B$ be a map of Spaces.

(1) If $\phi$ is an (acyclic) cofibration in $\operatorname{Fun}^{b}(\mathbf{A}, \mathcal{M})$, then $\mathbf{f}^{k} \phi$ is an (acyclic) cofibration in $\operatorname{Fun}^{b}(\mathbf{B}, \mathcal{M})$ [2, Theorem 11.2.]. In particular colim $\mathbf{A} \phi$ is an (acyclic) cofibration in $\mathcal{M}$.

(2) If $f$ is reduced and $\phi$ is an (acyclic) cofibration in $\operatorname{Fun}^{b}(\mathbf{B}, \mathcal{M})$, then so is $\mathbf{f}^{*} \phi$ in $\operatorname{Fun}^{b}(\mathbf{A}, \mathcal{M})$.

(3) If $\phi$ is an (acyclic) cofibration in $\operatorname{Fun}^{b}(\mathbf{N}(\mathbf{B}), \mathcal{M})$, then so is $\mathbf{N}(\mathbf{f})^{*} \phi$ in the category $\operatorname{Fun}^{b}(\mathbf{N}(\mathbf{A}), \mathcal{M})$.

The model structure given in Theorem 6.1 was introduced to study homotopy invariance of the colimit operation. The motivation was to explain the following Theorem.

Theorem 6.4 [2, Theorem 20.3, Corollary 21.4] Let $\phi: F \rightarrow G$ be a natural transformation in $\operatorname{Fun}^{b}(\mathbf{A}, \mathcal{M})$. If $\phi$ is a weak equivalence between cofibrant objects, then so is $\operatorname{colim}_{\mathbf{A}} \phi$.

Theorem 6.4 implies that the functor $\operatorname{colim}_{\mathbf{A}}: \operatorname{Fun}^{b}(\mathbf{A}, \mathcal{M}) \rightarrow \mathcal{M}$ has a left replacement (see 2.17) given by a cofibrant replacement $Q$ in $\operatorname{Fun}^{b}(\mathbf{A}, \mathcal{M})$. In particular its total left derived functor denoted by $\operatorname{ocolim}_{\mathbf{A}}: \operatorname{Fun}^{b}(\mathbf{A}, \mathcal{M}) \rightarrow \operatorname{Ho}(\mathcal{M})$ assigns to a bounded functor $F$ the object $\operatorname{colim}_{\mathbf{A}}(Q F)$ in $\mathcal{M}$ [2, Proposition 14.2]. The functor ocolim $\mathbf{A}$ coincides with hocolim ${ }_{\mathbf{A}\left[S^{-1}\right]}$ (the total left derived functor of $\left.\operatorname{colim}_{\mathbf{A}\left[S^{-1}\right]}: \operatorname{Fun}\left(\mathbf{A}\left[S^{-1}\right], \mathcal{M}\right) \rightarrow \mathcal{M}\right)$. In general ocolim $\mathbf{A}$ is not the restriction of the total left derived functor of $\operatorname{colim}_{\mathbf{A}}: \operatorname{Fun}(\mathbf{A}, \mathcal{M}) \rightarrow \mathcal{M}$. To construct hocolim $\mathbf{A}$ an additional step is necessary: $A$ needs to be subdivided first. This works in general for diagrams indexed by any small category $I$ and with values in any category that admits a left model approximation.

Theorem 6.5 [2, Theorem 11.3] Let $I$ be a small category, $\epsilon: \mathbf{N}(I) \rightarrow I$ the forgetful functor $\left(i_{n} \rightarrow \cdots \rightarrow i_{0}\right) \mapsto i_{0}$ and $l: \mathcal{M} \leftrightarrows \mathcal{C}: r$ a left model approximation. Then the following functors form a left model approximation:

$$
l \epsilon^{k}: \operatorname{Fun}^{b}(\mathbf{N}(I), \mathcal{M}) \rightleftarrows \operatorname{Fun}(I, \mathcal{C}): r \epsilon^{*} .
$$

Algebraic $8 \mathcal{G}$ Geometric Topology, Volume 8 (2008) 
Moreover, $F \mapsto l \epsilon^{k} Q\left(r \epsilon^{*} F\right)$ is a left replacement for $\operatorname{colim}_{I}: \operatorname{Fun}(I, \mathcal{C}) \rightarrow \mathcal{C}$.

Corollary 6.6 If $(\mathcal{C}, W)$ admits a left model approximation, then it can be left localized (see 2.18). In particular there is a functor $\otimes_{l}$ : Cat $\times \mathcal{C} \rightarrow \operatorname{Ho}(\mathcal{C})$ defined by the assignment $(I, X) \mapsto \operatorname{hocolim}_{I} c X$.

Proof According to Theorem 6.5, any functor $F: I \rightarrow \mathcal{C}$ can be modified functorially to $Q_{I} F:=l \epsilon^{k} Q\left(r \epsilon^{*} F\right)$ so that $\operatorname{colim}_{I} Q_{I} F$ represents $\operatorname{hocolim}_{I} F$ in $\operatorname{Ho}(\mathcal{C})$.

For categories admitting left model approximations, homotopy colimits can be characterized in an alternative way.

Corollary 6.7 Assume that $(\mathcal{C}, W)$ admits a left model approximation. The functor $\operatorname{hocolim}_{I}: \operatorname{Ho}(\operatorname{Fun}(I, \mathcal{C})) \rightarrow \operatorname{Ho}(\mathcal{C})$ is then left adjoint to the constant diagram functor $c: \operatorname{Ho}(\mathcal{C}) \rightarrow \operatorname{Ho}(\operatorname{Fun}(I, \mathcal{C}))$.

Proof If $\mathcal{M}$ is a model category, then $\operatorname{colim}_{\mathbf{K}}: \operatorname{Fun}^{b}(\mathbf{K}, \mathcal{M}) \rightarrow \mathcal{M}$ preserves (acyclic) cofibrations (Proposition 6.3). Thus by [8, Theorem 9.7], the total derived functors of the adjoint functors $\operatorname{colim}_{\mathbf{K}}: \operatorname{Fun}^{b}(\mathbf{K}, \mathcal{M}) \rightleftarrows \mathcal{M}: c$ also form an adjoint pair $\operatorname{ocolim}_{\mathbf{K}}: \operatorname{Ho}\left(\operatorname{Fun}^{b}(\mathbf{K}, \mathcal{M})\right) \rightleftarrows \operatorname{Ho}(\mathcal{M}): c$.

Choose a left model approximation $l: \mathcal{M} \leftrightarrows \mathcal{C}: r$. Let $F: I \rightarrow \mathcal{C}$ be a functor and $Y$ an object in $\mathcal{C}$. We have the following bijections between sets of morphisms respectively in $\operatorname{Ho}(\operatorname{Fun}(I, \mathcal{C})), \operatorname{Ho}\left(\operatorname{Fun}^{b}(\mathbf{N}(I), \mathcal{M})\right), \operatorname{Ho}(\mathcal{M})$, and $\operatorname{Ho}(\mathcal{C})$ :

$$
\begin{aligned}
{[F, c Y] } & \stackrel{(a)}{\cong}\left[r \epsilon^{*} F, r \epsilon^{*} c Y\right]=\left[r \epsilon^{*} F, c r Y\right] \stackrel{(b)}{\cong}\left[\operatorname{ocolim}_{\mathbf{N}(I)} r \epsilon^{*} F, r Y\right] \\
& \stackrel{(c)}{\cong}\left[L(l) \operatorname{ocolim}_{\mathbf{N}(I)} r \epsilon^{*} F, Y\right]=\left[\operatorname{hocolim}_{I} F, Y\right]
\end{aligned}
$$

where (a) follows from the fact that $l \epsilon^{k}: \operatorname{Fun}^{b}(\mathbf{N}(I), \mathcal{M}) \rightleftarrows \operatorname{Fun}(I, \mathcal{C}): r \epsilon^{*}$ is a left model approximation (Theorem 6.5) and [2, Proposition 5.5], (b) is a consequence of adjointness of $\operatorname{ocolim}_{\mathbf{N}(I)}$ and $c$, and the last bijection (c) follows from the following Lemma 6.8.

Lemma 6.8 If $l: \mathcal{M} \rightleftarrows \mathcal{C}: r$ is a left model approximation, then the total derived functors induce an adjoint pair $L(l): \operatorname{Ho}(\mathcal{M}) \rightleftarrows \operatorname{Ho}(\mathcal{C}): r$.

Proof The lemma is a consequence of two facts. First $r: \operatorname{Ho}(\mathcal{C}) \rightarrow \operatorname{Ho}(\mathcal{M})$ is a fullyfaithful embedding [2, Proposition 5.5]. Second $L(l)(r X) \rightarrow X$ is an isomorphism in $\operatorname{Ho}(\mathcal{C})$, for any $X$. 


\section{Constant functors}

Assume that $(\mathcal{C}, W)$ admits a left model approximation. To understand how this structure leads to an action of Spaces on $\mathcal{C}$ we are going to study properties of homotopy colimits of constant functors. The fundamental observation is the following proposition.

Proposition 7.1 If $f: I \rightarrow J$ is a weak equivalence of small categories, then, for any $X \in \mathcal{C}, f \otimes_{l} X: I \otimes_{l} X \rightarrow J \otimes_{l} X$ is an isomorphism in $\operatorname{Ho}(\mathcal{C})$.

Corollary 7.2 If $f: K \rightarrow L$ is a weak equivalence of spaces, then, for any $X \in \mathcal{C}$, $\mathbf{f} \otimes_{l} X: \mathbf{K} \otimes_{l} X \rightarrow \mathbf{L} \otimes_{l} X$ is an isomorphism in $\operatorname{Ho}(\mathcal{C})$.

Corollary 7.2 implies that the functor $(K, X) \mapsto$ hocolim $_{\mathbf{K}} c X=\mathbf{K} \otimes_{l} X$ is a composition of the localization Spaces $\times \mathcal{C} \rightarrow \operatorname{Ho}$ (Spaces) $\times \operatorname{Ho}(\mathcal{C})$ and a functor which we denote by the same symbol:

$$
\otimes_{l}: \operatorname{Ho}(\text { Spaces }) \times \operatorname{Ho}(\mathcal{C}) \rightarrow \operatorname{Ho}(\mathcal{C}) .
$$

The aim of this paper is to understand to what extent $\otimes_{l}$ is a homotopy action. We start by proving that it satisfies the first two requirements of 2.16 and will show in Proposition 9.4 that property (3) holds as well.

\section{Corollary 7.3}

(1) There are isomorphisms $[0] \otimes_{l} X \cong X$ in $\operatorname{Ho}(\mathcal{C})$ natural in $X \in \operatorname{Ho}(\mathcal{C})$.

(2) There are coherent isomorphisms $(\mathbf{K} \otimes \mathbf{L}) \otimes_{l} X \cong \mathbf{K} \otimes_{l}\left(\mathbf{L} \otimes_{l} X\right)$ in $\operatorname{Ho}(\mathcal{C})$ natural in $X \in \operatorname{Ho}(\mathcal{C})$ and $K, L \in \operatorname{Ho}$ (Spaces).

Proof Part (1) follows from Proposition 7.1 and the fact that the functor between the simplex category of $\Delta[0]$ and the category with only one object and one morphism is a weak equivalence of categories.

Recall that $\mathbf{K} \otimes \mathbf{L} \hookrightarrow \mathbf{K} \times \mathbf{L}$ is a weak equivalence of categories. Thus by Proposition 7.1, $(\mathbf{K} \otimes \mathbf{L}) \otimes_{l} X \rightarrow(\mathbf{K} \times \mathbf{L}) \otimes_{l} X$ is an isomorphism in $\operatorname{Ho}(\mathcal{C})$. According to [2, Theorem 24.9] there is a further isomorphism in $\operatorname{Ho}(\mathcal{C})$ :

$$
(\mathbf{K} \times \mathbf{L}) \otimes_{l} X=\operatorname{hocolim}_{\mathbf{K} \times \mathbf{L}} c X \cong \operatorname{hocolim}_{\mathbf{K}} \operatorname{hocolim}_{\mathbf{L}} c X=\mathbf{K} \otimes_{l}\left(\mathbf{L} \otimes_{l} X\right) .
$$

The coherence of these isomorphisms is straightforward. This concludes the proof. 
Proof of Proposition 7.1 Since $\epsilon: \mathbf{N}(I)^{\mathrm{op}} \rightarrow I, \quad\left(i_{n} \rightarrow \cdots \rightarrow i_{0}\right) \mapsto i_{n}$ is terminal [2, Theorem 29.1], the morphism hocolim $\mathrm{N}(I)^{\mathrm{op}} c X \rightarrow \operatorname{hocolim}_{I} c X$ is an isomorphism in $\operatorname{Ho}(\mathcal{C})$ for any $X \in \mathcal{C}$. To prove the proposition it remains to show that a weak equivalence of spaces $f: K \rightarrow L$ induces an isomorphism $\mathbf{f}^{\text {op }} \otimes_{l} X: \mathbf{K}^{\mathrm{op}} \otimes_{l} X \rightarrow$ $\mathbf{L}^{\text {op }} \otimes_{l} X$ in $\operatorname{Ho}(\mathcal{C})$.

Let $\mathbf{S}$ be the collection of maps of spaces $f: K \rightarrow L$ such that, for any category $(\mathcal{C}, W)$ admitting a left model approximation and for any $X \in \mathcal{C}, \mathbf{f}^{\text {op }} \otimes_{l} X$ is an isomorphism in $\operatorname{Ho}(\mathcal{C})$. We claim that:

(1) If $K$ and $L$ are contractible, then any $f: K \rightarrow L$ belongs to $\mathbf{S}$.

(2) Maps of the form $\Delta[n, k] \hookrightarrow \Delta[n]$ belong to $\mathbf{S}$.

(3) $\mathbf{S}$ is closed under retracts.

(4) $\mathbf{S}$ is closed under arbitrary sums.

(5) If an inclusion $f: K \hookrightarrow L$ belongs to $\mathbf{S}$, then, for any map $K \rightarrow A$, so does the inclusion $A \hookrightarrow \operatorname{colim}(A \leftarrow K \stackrel{f}{\hookrightarrow} L)$.

(6) If a sequence of inclusions $K_{i} \hookrightarrow K_{i+1}(i>0)$ belong to $\mathbf{S}$, then so does $K_{1} \hookrightarrow \operatorname{colim}_{i>0} K_{i}$.

(7) A one-sided inverse of an element in $\mathbf{S}$ belongs to $\mathbf{S}$.

Note that properties (2)-(6) imply that any trivial cofibration of spaces belongs to $\mathbf{S}$. And since any weak equivalence can be factored as a trivial cofibration followed by a left inverse of a trivial cofibration, the proposition clearly follows from property (7). Let us thus prove the claim.

Property (1) is a consequence of [2, Corollary 29.2] and (2) is a particular case of it. Property (3) is obvious and property (7) follows from a "two-out-of-three argument". Since the arguments for (4), (5), and (6) are basically the same, we present the details of how to prove (5) only.

Set $D$ to be $\operatorname{colim}(A \leftarrow K \stackrel{f}{\hookrightarrow} L)$ and fix a left model approximation $l: \mathcal{M} \rightleftarrows \mathcal{C}: r$. For an object $X \in \mathcal{C}$, choose a cofibrant replacement $Q X$ in $\operatorname{Fun}^{b}\left(N\left(\mathbf{D}^{\mathrm{op}}\right), \mathcal{M}\right)$ of the constant diagram $\operatorname{cr} X$. Consider next the following push-out square of spaces:

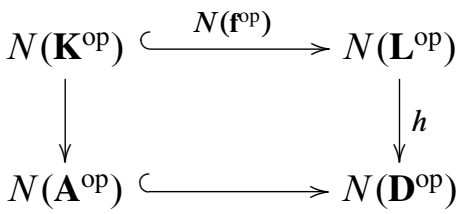

Algebraic 83 Geometric Topology, Volume 8 (2008) 
Note that all the maps in this diagram are reduced and the horizontal maps are cofibrations. It follows that $h^{*} Q X$ is cofibrant in $\operatorname{Fun}^{b}\left(\mathbf{N}\left(\mathbf{L}^{\text {op }}\right), \mathcal{M}\right)$ and consequently the morphism $\operatorname{colim}_{\mathbf{N}\left(\mathbf{K}^{\text {op }}\right)} Q X \rightarrow \operatorname{colim}_{\mathbf{N}\left(\mathbf{L}^{\text {op }}\right)} Q X$ is a cofibration (Proposition 6.2). Moreover it is an acyclic cofibration as $f$ is assumed to be in $\mathbf{S}$. This shows that $\operatorname{colim}_{\mathbf{N}\left(\mathbf{A}^{\mathrm{op}}\right)} Q X \rightarrow \operatorname{colim}_{\mathbf{N}\left(\mathbf{D}^{\text {op }}\right)} Q X$ is also an acyclic cofibration between cofibrant objects. The morphism $l\left(\operatorname{colim}_{\mathbf{N}\left(\mathbf{A}^{\text {op }}\right)} Q X\right) \rightarrow l\left(\operatorname{colim}_{\mathbf{N}\left(\mathbf{D}^{\text {op }}\right)} Q X\right)$ is therefore a weak equivalence in $\mathcal{C}$. Since it can be identified with $\mathbf{A}^{\mathrm{op}} \otimes_{l} X \rightarrow \mathbf{D}^{\mathrm{op}} \otimes_{l} X$, the claim is proven.

\section{An almost simplicial model structure}

Let $\mathcal{M}$ be a model category and $A$ be a space. The category $\operatorname{Fun}^{b}(\mathbf{N}(\mathbf{A}), \mathcal{M})$ has both a model structure (Theorem 6.1) and a simplicial structure (Proposition 5.3). How compatible are they? To what extent is it a simplicial model category? How related are the functors:

$$
\begin{aligned}
\otimes_{l}: \text { Spaces } \times \operatorname{Fun}^{b}(\mathbf{N}(\mathbf{A}), \mathcal{M}) & \rightarrow \operatorname{Ho}\left(\operatorname{Fun}^{b}(\mathbf{N}(\mathbf{A}), \mathcal{M})\right) \\
\otimes: \text { Spaces } \times \operatorname{Fun}^{b}(\mathbf{N}(\mathbf{A}), \mathcal{M}) & \rightarrow \operatorname{Fun}^{b}(\mathbf{N}(\mathbf{A}), \mathcal{M}) ?
\end{aligned}
$$

Example 8.1 Let us choose a simplex $\sigma: \Delta[0] \rightarrow N(\mathbf{A})$. By definition it is given by an object in $\mathbf{A}$, ie, a map $\Delta[n] \rightarrow A$. Let $F \in \operatorname{Fun}^{b}(\mathbf{N}(\mathbf{A})$, Spaces $)$ be a cofibrant diagram such that $F(\sigma) \neq \varnothing$. Note that:

$$
\lim (\Delta[0] \stackrel{\sigma}{\rightarrow} N(\mathbf{A}) \stackrel{\pi}{\leftarrow} N([1] \otimes \mathbf{A}))=\coprod_{\operatorname{mor}([n],[1])} \Delta[0] .
$$

Therefore $(\Delta[1] \otimes F)(\sigma)=\pi^{k} \pi^{*} F=\bigsqcup_{\operatorname{mor}([n],[1])} F(\sigma)$ which is not weakly equivalent to $F(\sigma)$. It follows that the map $\Delta[1] \rightarrow \Delta[0]$ does not induce a weak equivalence of functors $\Delta[1] \otimes F \rightarrow \Delta[0] \otimes F=F$.

Thus the operation $-\otimes F$ does not preserve weak equivalences, and consequently Fun $^{b}(\mathbf{N}(\mathbf{A})$, Spaces $)$ is not a simplicial model category. Further, since the functor $-\otimes_{l} F$ preserves weak equivalences (see Corollary 7.2), the objects $\Delta[1] \otimes F$ and $[1] \otimes_{l} F$ are not isomorphic in $\operatorname{Ho}_{\left(F_{n}\right.}^{b}(\mathbf{N}(\mathbf{A})$, Spaces $\left.)\right)$.

Despite this negative example, we are going to show that only half of Quillen's axiom (SM7) [16] does not hold for $\mathrm{Fun}^{b}(\mathbf{N}(\mathbf{A}), \mathcal{M})$ to be a simplicial model category. Moreover we are going to prove that, for any homotopically constant and cofibrant $F \in \operatorname{Fun}^{b}(\mathbf{N}(\mathbf{A}), \mathcal{M}), \operatorname{colim}_{\mathbf{N}(\mathbf{A})}(K \otimes F)$ and $\operatorname{ocolim}_{\mathbf{N}(\mathbf{A})}\left(\mathbf{K} \otimes_{l} F\right)$ (see the remark 
after Theorem 6.4) are isomorphic in $\operatorname{Ho}(\mathcal{M})$, although $K \otimes F$ and $\mathbf{K} \otimes_{l} F$ might fail to be so in $\left.\operatorname{Ho}_{\left(\operatorname{Fun}^{b}\right.}(\mathbf{N}(\mathbf{A}), \mathcal{M})\right)$.

Let $\phi: F \rightarrow G$ be a morphism in $\operatorname{Fun}^{b}(\mathbf{N}(\mathbf{A}), \mathcal{M})$ and $f: K \rightarrow L$ be a map of spaces. Define:

$$
H:=\operatorname{colim}(L \otimes F \stackrel{f \otimes F}{\longleftarrow} K \otimes F \stackrel{K \otimes \phi}{\longrightarrow} K \otimes G) .
$$

Let $\psi: H \rightarrow L \otimes G$ in $\operatorname{Fun}^{b}(\mathbf{N}(\mathbf{A}), \mathcal{M})$ be induced by $f, \phi$, and the universal property of the colimit. The following proposition shows that half of Quillen's axiom (SM7) [16] holds for the simplicial structure introduced in Section 5.

Proposition 8.2 If $f: K \hookrightarrow L$ is an inclusion of spaces and $\phi: F \rightarrow G$ is an (acyclic) cofibration in $\mathrm{Fun}^{b}(\mathbf{N}(\mathbf{A}), \mathcal{M})$, then $\psi: H \rightarrow L \otimes G$ is an (acyclic) cofibration in $\operatorname{Fun}^{b}(\mathbf{N}(\mathbf{A}), \mathcal{M})$.

Proof We need to show that, for a non-degenerate $\sigma: \Delta[n] \rightarrow N(\mathbf{A})$, the morphism:

$$
i: \operatorname{colim}\left(H(\sigma) \leftarrow \operatorname{colim}_{\partial \Delta[n]} H \rightarrow \operatorname{colim}_{\partial \Delta[n]}(L \otimes G)\right) \longrightarrow(L \otimes G)(\sigma)
$$

is an (acyclic) cofibration in $\mathcal{M}$ (Theorem 6.1). To identify both sides of this morphism consider the following commutative diagram, where all the squares are pull-backs:

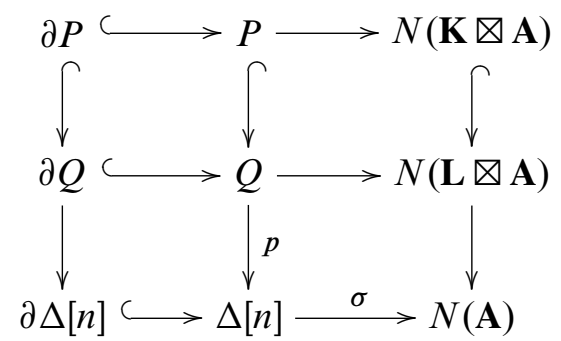

All the vertical maps in this diagram are reduced, as they are pull-backs of reduced maps (Proposition 5.1). Moreover, since $\partial Q \hookrightarrow Q$ and $P \hookrightarrow Q$ are inclusions, the map $R:=\operatorname{colim}(\partial Q \leftarrow \partial P \rightarrow P) \rightarrow Q$ is a monomorphism. The fact that $p: Q \rightarrow \Delta[n]$ is reduced, implies that the only non-degenerate simplices in $Q \backslash \partial Q$ are of dimension $n$ and $p$ sends them to the only non-degenerate simplex of dimension $n$ in $\Delta[n]$. As $\sigma$ is non degenerate in $N(\mathbf{A})$ and $(Q \backslash R) \subset(Q \backslash \partial Q)$, it follows that the composition $\sigma p$ takes non-degenerate simplices in $Q \backslash R$ to non-degenerate simplices in $N(\mathbf{A})$.

The spaces in this diagram can be used to make the following identifications:

$$
\begin{array}{ll}
\operatorname{colim}_{\partial \boldsymbol{\Delta}[n]}(L \otimes F)=\operatorname{colim}_{\partial \boldsymbol{Q}} F & (L \otimes F)(\sigma)=\operatorname{colim}_{\mathbf{Q}} F \\
\operatorname{colim}_{\partial \boldsymbol{\Delta}[n]}(L \otimes G)=\operatorname{colim}_{\partial \boldsymbol{Q}} G & (L \otimes G)(\sigma)=\operatorname{colim}_{\mathbf{Q}} G \\
\operatorname{colim}_{\partial \boldsymbol{\Delta}[n]}(K \otimes F)=\operatorname{colim}_{\partial} F & (K \otimes F)(\sigma)=\operatorname{colim}_{\mathbf{P}} F \\
\operatorname{colim}_{\partial \boldsymbol{\Delta}[n]}(K \otimes G)=\operatorname{colim}_{\partial \boldsymbol{P}} G & (K \otimes G)(\sigma)=\operatorname{colim}_{\mathbf{P}} G .
\end{array}
$$


Thus the left hand side of the morphism $i$ coincides with the colimit of the cube:

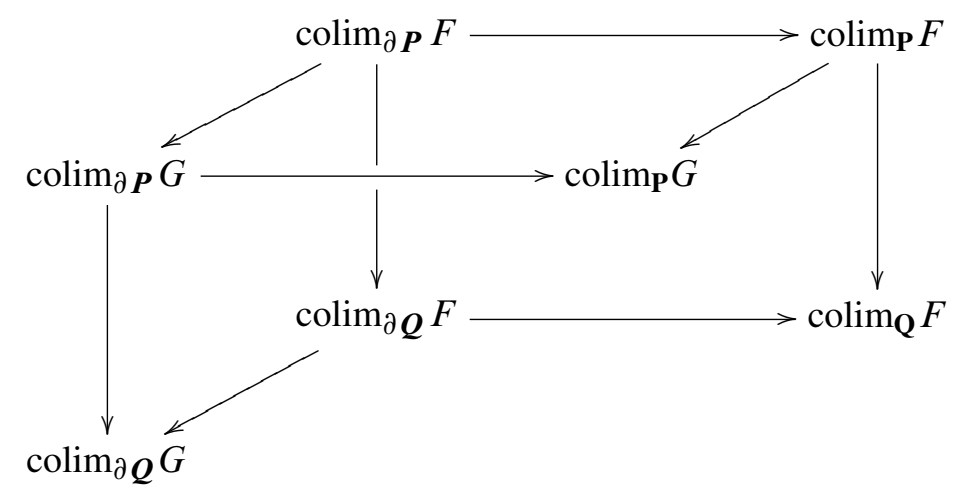

Note that:

$$
\begin{aligned}
& \operatorname{colim}\left(\operatorname{colim}_{\partial \boldsymbol{Q}} F \leftarrow \operatorname{colim}_{\partial \boldsymbol{P}} F \rightarrow \operatorname{colim}_{\mathbf{P}} F\right)=\operatorname{colim}_{\mathbf{R}} F \\
& \operatorname{colim}\left(\operatorname{colim}_{\partial \boldsymbol{Q}} G \leftarrow \operatorname{colim}_{\partial \boldsymbol{P}} G \rightarrow \operatorname{colim}_{\mathbf{P}} G\right)=\operatorname{colim}_{\mathbf{R}} G .
\end{aligned}
$$

Thomason's [2, Theorem 26.8] permits in general to decompose the (ho)colimit over a Grothendieck construction into simpler (ho)colimits. In the particular case of a cube as above, [2, Example 38.2] shows that the colimit can be also expressed as a pushout (see also Goodwillie [9, Claim 2.8]):

$$
\operatorname{colim}\left(\operatorname{colim}_{\mathbf{Q}} F \leftarrow \operatorname{colim}_{\mathbf{R}} F \hookrightarrow \operatorname{colim}_{\mathbf{R}} G\right)
$$

Moreover $i$ can be identified with the morphism induced by the inclusion $R \subset Q$ and the natural transformation $\phi: F \rightarrow G$ :

$$
\operatorname{colim}\left(\operatorname{colim}_{\mathbf{Q}} F \leftarrow \operatorname{colim}_{\mathbf{R}} F \hookrightarrow \operatorname{colim}_{\mathbf{R}} G\right) \rightarrow \operatorname{colim}_{\mathbf{Q}} G .
$$

We can now apply Proposition 6.2 to conclude that this morphism is an (acyclic) cofibration in $\mathcal{M}$.

Although in general the operation $K \mapsto K \otimes F$ is not homotopy invariant (Example 8.1 ), it behaves well on homotopically constant diagrams.

Proposition 8.3 Let $F \in F_{u n}^{b}(\mathbf{N}(\mathbf{A}), \mathcal{M})$ be homotopically constant, 2.4, and cofibrant. Then:

(1) For any a weak equivalence of spaces $f: K \rightarrow L$, the induced morphism $\operatorname{colim}_{\mathbf{N}(\mathbf{A})}(f \otimes F): \operatorname{colim}_{\mathbf{N}(\mathbf{A})}(K \otimes F) \rightarrow \operatorname{colim}_{\mathbf{N}(\mathbf{A})}(L \otimes F)$ is a weak equivalence in $\mathcal{M}$. 
(2) There is an isomorphism $\operatorname{colim}_{\mathbf{N}(\mathbf{A})}(K \otimes F) \cong \operatorname{ocolim}_{\mathbf{N}(\mathbf{A})}\left(\mathbf{K} \otimes_{l} F\right)$ in $\operatorname{Ho}(\mathcal{M})$, for any space $K$.

Proof Choose a weak equivalence $F \rightarrow c X$ in $\operatorname{Fun}^{b}(\mathbf{N}(\mathbf{A}), \mathcal{M})$. Let $\pi$ be the subdivision of the projection $K \times A \rightarrow A$ and note that there is an isomorphism $\operatorname{colim}_{\mathbf{N}(\mathbf{A})}(K \otimes F) \cong \operatorname{colim}_{\mathbf{N}(\mathbf{K} \otimes \mathbf{A})} \pi^{*} F$. Since $\pi$ is reduced, the bounded diagram $\pi^{*} F$ is cofibrant in $\operatorname{Fun}^{b}(\mathbf{N}(\mathbf{K} \otimes \mathbf{A}), \mathcal{M})$. It follows that there is an isomorphism $\operatorname{colim}_{\mathbf{N}(\mathbf{A})}(K \otimes F) \cong(\mathbf{K} \otimes \mathbf{A}) \otimes_{l} X$ in $\operatorname{Ho}(\mathcal{M})$. By the same argument, the morphism $(\mathbf{f} \otimes \mathbf{A}) \otimes_{l} X:(\mathbf{K} \otimes \mathbf{A}) \otimes_{l} X \rightarrow(\mathbf{L} \otimes \mathbf{A}) \otimes_{l} X$ can be identified with colim $\operatorname{N}_{\mathbf{N}(\mathbf{A})}(f \otimes F)$. Since $f \times A$ is a weak equivalences, according to Corollary 7.2, $(\mathbf{f} \otimes \mathbf{A}) \otimes_{l} X$ is an isomorphism in $\operatorname{Ho}(\mathcal{M})$ and so $\operatorname{colim}_{\mathbf{N}(\mathbf{A})}(f \otimes F)$ is a weak equivalence in $\mathcal{M}$. This shows the first statement.

To prove the second statement consider the following sequence of isomorphisms in $\operatorname{Ho}(\mathcal{M})$ :

$$
\begin{aligned}
\operatorname{colim}_{\mathbf{N}(\mathbf{A})}(K \otimes F) & \cong(\mathbf{K} \otimes \mathbf{A}) \otimes_{l} X \stackrel{(a)}{\cong}(\mathbf{K} \times \mathbf{A}) \otimes_{l} X \stackrel{(b)}{\cong}(\mathbf{A} \times \mathbf{K}) \otimes_{l} X \\
& \stackrel{(c)}{\cong} \mathbf{A} \otimes_{l}\left(\mathbf{K} \otimes_{l} X\right) \cong \operatorname{ocolim}_{\mathbf{N}(\mathbf{A})}\left(\mathbf{K} \otimes_{l} F\right)
\end{aligned}
$$

where $(a)$ and $(b)$ are isomorphisms because of Corollary 7.2 and $(c)$ is an isomorphism because of [2, Theorem 24.9].

How do these properties of the operation $\otimes$ in $\operatorname{Fun}^{b}(\mathbf{N}(\mathbf{A}), \mathcal{M})$ translate to the mapping spaces introduced in Proposition 5.3?

Corollary 8.4 If $\phi$ is a weak equivalence between cofibrant objects and $\psi$ is a weak equivalence between fibrant objects in $\operatorname{Fun}^{b}(\mathbf{N}(\mathbf{A}), \mathcal{M})$, then map $(\phi, \psi)$ is a weak equivalence of spaces.

Proof By standard K Brown lemma type of arguments [8, Lemma 9.9], without loss of generality, we can assume in addition that $\phi$ is an acyclic cofibration and $\psi$ is an acyclic fibration. In this case the corollary follows from Proposition 8.2 and the usual adjointness argument.

The mapping space functor satisfies also the following duality property.

Proposition 8.5 If $H \in \operatorname{Fun}^{b}\left(\mathbf{K}, \operatorname{Fun}^{b}(\mathbf{N}(\mathbf{A}), \mathcal{M})\right)$ is a cofibrant functor whose values are homotopically constant functors in $\operatorname{Fun}^{b}(\mathbf{N}(\mathbf{A}), \mathcal{M})$ and $Z$ is fibrant in $\mathcal{M}$, then $\operatorname{map}(H, c Z)$ is fibrant in $\operatorname{Fun}^{b}\left(\mathbf{K}^{\mathrm{op}}\right.$, Spaces) [2, Section 31.3]. 
Proof We need to show that, for a non-degenerate simplex $\sigma: \Delta[n] \rightarrow K$, we have a fibration of spaces

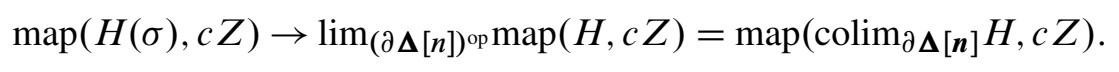

To do that one needs to construct certain lifts. By adjointness this problem reduces to showing that, for a push-out square of the form:

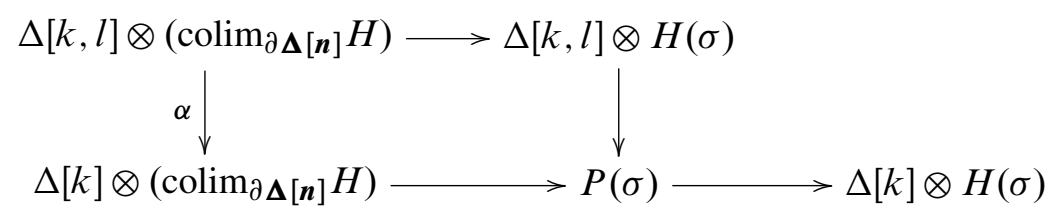

any natural transformation $P \rightarrow c Z$ factors through $P \rightarrow \Delta[k] \otimes H(-)$. Since $c Z$ is a constant functor and $Z$ is fibrant, it is enough to prove that the morphism $\operatorname{colim}_{\mathbf{N}(\mathbf{A})} P \rightarrow \operatorname{colim}_{\mathbf{N}(\mathbf{A})}(\Delta[k] \otimes H(\sigma))$ is an acyclic cofibration in $\mathcal{M}$. Cofibrancy is a consequence of Proposition 8.2 and Proposition 6.3.(1). Using Proposition 8.3, acyclicity would follow if $\operatorname{colim}_{\mathbf{N}(\mathbf{A})} \alpha$ is an acyclic cofibration in $\mathcal{M}$. Since $H$ is cofibrant, the same is true for $\operatorname{colim}_{\partial \boldsymbol{\Delta}[\boldsymbol{n}]} H$ and so $\alpha$ is a cofibration by Proposition 8.2. This implies that $\operatorname{colim}_{\mathbf{N}(\mathbf{A})} \alpha$ is also a cofibration. It remains to show that $\operatorname{colim}_{\mathbf{N}(\mathbf{A})} \alpha$ is a weak equivalence.

Note that, for any $L, L \otimes H$ is cofibrant in $\operatorname{Fun}^{b}\left(\mathbf{K}, \operatorname{Fun}^{b}(\mathbf{N}(\mathbf{A}), \mathcal{M})\right)$ (another consequence of Proposition 8.2). The values of $H$ are cofibrant and homotopically constant. We can therefore apply Proposition 8.3 to conclude that the natural transformation $\operatorname{colim}_{\mathbf{N}(\mathbf{A})}(\Delta[k, l] \otimes H) \rightarrow \operatorname{colim}_{\mathbf{N}(\mathbf{A})}(\Delta[k] \otimes H)$ is a weak equivalence between cofibrant objects in $\operatorname{Fun}^{b}(\mathbf{K}, \mathcal{M})$ [2, Proposition 24.2]. Consequently $\operatorname{colim}_{\mathbf{K}} \operatorname{colim}_{\mathbf{N}(\mathbf{A})}(\Delta[k, l] \otimes H) \rightarrow \operatorname{colim}_{\mathbf{K}} \operatorname{colim}_{\mathbf{N}(\mathbf{A})}(\Delta[k] \otimes H)$ is a weak equivalence in $\mathcal{M}$. As this last morphism can be identified with $\operatorname{colim}_{\mathbf{N}(\mathbf{A})} \alpha$, the proposition is proven.

This implies that the connected components of certain mapping spaces coincide as expected with homotopy classes of morphisms.

Corollary 8.6 If $Z$ is fibrant in $\mathcal{M}$ and $F$ is homotopically constant and cofibrant in $\operatorname{Fun}^{b}(\mathbf{N}(\mathbf{A}), \mathcal{M})$, then $\operatorname{map}(F, c Z)$ is Kan. Moreover there is a bijection, natural in $F$ and $Z$, between the set of components $\pi_{0} \operatorname{map}(F, c Z)$ and the set of morphism $[F, c Z]$ in $\left.\operatorname{Ho}_{\left(\operatorname{Fun}^{b}\right.}(\mathbf{N}(\mathbf{A}), \mathcal{M})\right)$.

Proof The first part is a particular case of Proposition 8.5 when $K=\Delta[0]$. To prove the second part, we will show that the map which assigns to the component of 
a morphism $\phi: F \rightarrow c Z$ in $\operatorname{map}(F, c Z)$ the homotopy class of $\phi$ in $[F, c Z]$, is a bijection.

Since $F$ is cofibrant, the natural transformation $\partial \Delta[1] \otimes F \rightarrow \Delta[1] \otimes F$, induced by the inclusion $\partial \Delta[1] \hookrightarrow \Delta[1]$, is a cofibration. Consider two natural transformations $\phi_{0}: F \rightarrow c Z$ and $\phi_{1}: F \rightarrow c Z$, choose a cylinder object $H$ for $F$, and consider the following commutative diagram:

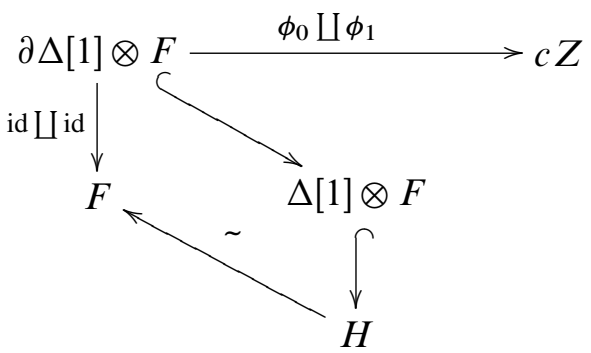

We claim that there is a natural transformation $\Delta[1] \otimes F \rightarrow c Z$ which makes the above diagram commutative if and only if there is a natural transformation $H \rightarrow c Z$ with the same property. Since the target $c Z$ is a constant functor any such map $\Delta[1] \otimes F \rightarrow c Z$ is induced by a morphism colim $\operatorname{N(A)}_{(A}(\Delta[1] \otimes F) \rightarrow Z$ in $\mathcal{M}$. From Proposition 8.3 and Proposition 6.3(1) we infer that the morphisms $\operatorname{colim}_{\mathbf{N}(\mathbf{A})}(\Delta[1] \otimes F) \rightarrow \operatorname{colim}_{\mathbf{N}(\mathbf{A})} F$ and $\operatorname{colim}_{\mathbf{N}(\mathbf{A})} H \rightarrow \operatorname{colim}_{\mathbf{N}(\mathbf{A})} F$ are weak equivalences. It follows that the morphism $\operatorname{colim}_{\mathbf{N}(\mathbf{A})}(\Delta[1] \otimes F) \rightarrow \operatorname{colim}_{\mathbf{N}(\mathbf{A})} H$ is an acyclic cofibration in $\mathcal{M}$ and our claim is proven, as $Z$ is assumed to be fibrant. This argument shows that $\phi_{0}$ and $\phi_{1}$ are left homotopic if and only if they are in the same component of map $(F, c Z)$.

\section{Resolutions and mapping spaces}

A standard way of studying derived or homotopical properties of various operations is by considering resolutions. This resolution principle is playing a significant role in our approach to mapping spaces too. In our setting it is stated as a model approximation.

Proposition 9.1 Let $\mathcal{M}$ be a model category. If $A$ is a contractible space, then the pair of adjoint functors colim $\mathbf{N}(\mathbf{A}): \operatorname{Fun}^{b}(\mathbf{N}(\mathbf{A}), \mathcal{M}) \rightleftarrows \mathcal{M}: c$ is a left model approximation of $\mathcal{M}$.

Proof Conditions (1), (2), and (3) of Definition 1.1 are obviously satisfied. Condition (4) is a consequence of Theorem 6.4. To prove (5) consider a weak equivalence $F \rightarrow c X$ in $\operatorname{Fun}^{b}(\mathbf{N}(\mathbf{A}), \mathcal{M})$ with a cofibrant domain. According to Theorem 6.5, colim $\mathbf{N}_{\mathbf{N}(\mathbf{A})} F$ is isomorphic to $\mathbf{A} \otimes_{l} X$ in $\operatorname{Ho}(\mathcal{M})$. Since $A$ is contractible, the functor from the 
simplex category of $A$ to the category with only one object and one morphism is a weak equivalence. Thus, by Proposition 7.1, $\mathbf{A} \otimes_{l} X \rightarrow X$ is also an isomorphism in $\mathrm{Ho}(\mathcal{M})$ and so $\operatorname{colim}_{\mathbf{N}(\mathbf{A})} F \rightarrow X$ is a weak equivalence in $\mathcal{M}$.

Taking the subdivision of $A$ is a crucial assumption in the statement of Proposition 9.1. It is not true in general that if $A$ is contractible, then the pair of adjoint functors $\operatorname{colim}_{\mathbf{A}}: \operatorname{Fun}^{b}(\mathbf{A}, \mathcal{M}) \rightleftarrows \mathcal{M}: c$ forms a left model approximation. Take $\mathcal{M}$ to be Spaces and $A$ to be the space defined in Example 4.1. Consider a weak equivalence $F \rightarrow c \Delta[0]$ in $\operatorname{Fun}^{b}\left(\mathbf{A}\right.$, Spaces) with a cofibrant domain. The space $\operatorname{colim}_{\mathbf{A}} F$ is weakly equivalent to hocolim ${ }_{\mathrm{A}\left[S^{-1}\right]} \Delta[0] \simeq S^{2}$, which is not weakly contractible. Thus the requirement (5) of Definition 1.1 is not satisfied.

How to construct mapping spaces in a category $\mathcal{C}$ that admits a left model approximation? Here is the recipe:

- choose a left model approximation $l: \mathcal{M} \rightleftarrows \mathcal{C}: r$;

- choose a contractible space $A$;

- choose a cofibrant replacement $Q$ in $\operatorname{Fun}^{b}(\mathbf{N}(\mathbf{A}), \mathcal{M})$ and a fibrant replacement $R$ in $\mathcal{M}$;

- define a functor map: $\mathcal{C}^{\mathrm{op}} \times \mathcal{C} \rightarrow$ Spaces by the formula:

$$
\operatorname{map}(X, Y):=\operatorname{map}(\operatorname{Qcr}(X), c \operatorname{Rr}(Y))
$$

where the mapping space on the right comes from the simplicial structure on $\operatorname{Fun}^{b}(\mathbf{N}(\mathbf{A}), \mathcal{M})$ introduced in Proposition 5.3.

These mapping spaces have the following properties.

\section{Proposition 9.2}

(1) For any $X$ and $Y$ in $\mathcal{C}, \operatorname{map}(X, Y)$ is Kan.

(2) There is a bijection, natural in $X$ and $Y$ in $\mathcal{C}$, between the set of connected components $\pi_{0} \operatorname{map}(X, Y)$ and the set of morphism $[X, Y]$ in $\operatorname{Ho}(\mathcal{C})$.

(3) If $\alpha: X_{0} \rightarrow X_{1}$ and $\beta: Y_{0} \rightarrow Y_{1}$ are weak equivalences in $\mathcal{C}$, then

$$
\operatorname{map}(\alpha, \beta): \operatorname{map}\left(X_{1}, Y_{0}\right) \rightarrow \operatorname{map}\left(X_{0}, Y_{1}\right)
$$

is a weak equivalence of spaces. 
Proof Statement (1) is a direct consequence of the first part of Corollary 8.6.

According to the second part of this corollary there is a natural bijection between the set of connected components $\pi_{0} \operatorname{map}(X, Y)$ and the set of morphism $[\operatorname{cr}(X), \operatorname{cr}(Y)]$ in $\operatorname{Ho}\left(\operatorname{Fun}^{b}(\mathbf{N}(\mathbf{A}), \mathcal{M})\right)$. Since $A$ is contractible, Proposition 9.1 implies that the pair $l \operatorname{colim}_{\mathbf{N}(\mathbf{A})}: \operatorname{Fun}^{b}(\mathbf{N}(\mathbf{A}), \mathcal{M}) \rightleftarrows \mathcal{C}: c r$ forms a left model approximation of $\mathcal{C}$. It follows that there is a bijection between the set of morphisms $[X, Y]$ in $\operatorname{Ho}(\mathcal{C})$ and $[\operatorname{cr}(X), \operatorname{cr}(Y)]$ in $\left.\operatorname{Ho}_{\left(\operatorname{Fun}^{b}\right.}(\mathbf{N}(\mathbf{A}), \mathcal{M})\right)$ [2, Proposition 5.5], which shows (2).

Statement (3) is a direct consequence of Corollary 8.4.

Remark 9.3 Assume that, in the above recipe for constructing mapping spaces, we chose a model category $\mathcal{M}$ which has a functorial cofibrant-fibrant replacement. This induces in turn a functorial cofibrant-fibrant replacement in $\operatorname{Fun}^{b}(\mathbf{N}(\mathbf{A}), \mathcal{M})$. For any $X$ in $\mathcal{C}$ define $\widetilde{X}$ to be this replacement for the constant functor $\operatorname{cr}(X)$. Such choice leads not only to a functor $\operatorname{map}(X, Y):=\operatorname{map}(\widetilde{X}, \widetilde{Y})$, but also to a strictly associative composition

$$
\operatorname{map}(X, Y) \times \operatorname{map}(Y, Z) \longrightarrow \operatorname{map}(X, Z)
$$

which comes from the simplicial enrichment introduced in Proposition 5.3.

Part (3) of Proposition 9.2 implies that the mapping space functor induces a functor on the level of homotopy categories, denoted by the same symbol:

$$
\text { map: } \operatorname{Ho}(\mathcal{C})^{\text {op }} \times \operatorname{Ho}(\mathcal{C}) \rightarrow \operatorname{Ho}(\text { Spaces }) .
$$

How dependent is this functor on the choices we have made: the model approximation $l: \mathcal{M} \rightleftarrows \mathcal{C}: r$, the contractible space $A$, and the cofibrant and fibrant replacements? It can be characterized by the following universal property.

Proposition 9.4 Let $X \in \mathcal{C}$. The functor $\operatorname{map}(X,-)$ : $\mathrm{Ho}(\mathcal{C}) \rightarrow \operatorname{Ho}($ Spaces $)$ is right adjoint to $-\otimes_{l} X$ : $\operatorname{Ho}($ Spaces $) \rightarrow \operatorname{Ho}(\mathcal{C})$. In particular it does not depend on the choices of the model approximation $l: \mathcal{M} \rightleftarrows \mathcal{C}: r$, the contractible space $A$, and the cofibrant and fibrant replacements.

Proof Consider the following sequence of adjoint functors:

$$
\text { Spaces } \underset{\operatorname{map}(Q c r X,-)}{\stackrel{-\otimes Q c r X}{\rightleftarrows}} \operatorname{Fun}^{b}(\mathbf{N}(\mathbf{A}), \mathcal{M}) \underset{c}{\stackrel{\operatorname{colim}_{\mathbf{N}(\mathbf{A})}}{\rightleftarrows}} \mathcal{M} \underset{r}{\stackrel{l}{\rightleftarrows}} \mathcal{C} \text {. }
$$


According to Propositions 6.3, 8.2, and 8.3, the functor colim $\operatorname{Na}_{\mathbf{N}(\mathbf{A})}(-\otimes \operatorname{Qcr} X)$ preserves cofibrations and acyclic cofibrations. Thus, by [8, Theorem 9.7], the total derived functors also form adjoint pairs (see also Lemma 6.8):

$$
\mathrm{Ho}(\text { Spaces }) \underset{R(\operatorname{map}(Q c r X, c-))}{\frac{L\left(\operatorname{colim}_{\mathbf{N}(\mathbf{A})}(-\otimes Q c r X)\right)}{\rightleftarrows}} \operatorname{Ho}(\mathcal{M}) \stackrel{L(l)}{\underset{r}{\rightleftarrows}} \operatorname{Ho}(\mathcal{C}) .
$$

Note that the composition of $L\left(\operatorname{colim}_{\mathbf{N}(\mathbf{A})}(-\otimes Q \operatorname{cr} X)\right)$ and $L(l)$ can be identified with $K \mapsto(\mathbf{K} \otimes \mathbf{A}) \otimes_{l} X$ (see the proof of Proposition 8.3), and the composition of $r$ and $R(\operatorname{map}(Q c r X, c-))$ with $\operatorname{map}(X,-): \operatorname{Ho}(\mathcal{C}) \rightarrow \operatorname{Ho}$ (Spaces). Since $A$ is assumed to be contractible, the morphism $(\mathbf{K} \otimes \mathbf{A}) \otimes_{l} X \rightarrow \mathbf{K} \otimes_{l} X$, induced by the projection $\pi: K \times A \rightarrow K$, is an isomorphism in $\operatorname{Ho}(\mathcal{C})$ (Corollary 7.2).

Corollary 9.5 Assume that $(\mathcal{C}, W)$ admits a left model approximation. The functor $\otimes_{l}: \operatorname{Ho}($ Spaces $) \times \operatorname{Ho}(\mathcal{C}) \rightarrow \operatorname{Ho}(\mathcal{C})$ is then a homotopy left action 2.15 .

How natural is this homotopy left action? Let $(\mathcal{C}, W)$ and $(\mathcal{D}, V)$ admit left model approximations and $\Phi: \mathcal{C} \rightarrow \mathcal{D}$ be a functor that sends weak equivalences in $\mathcal{C}$ to weak equivalences in $\mathcal{D}$. The universal property of the total left derived functor hocolim $I$ yields a morphism hocolim $I \Phi \rightarrow \Phi\left(\operatorname{hocolim}_{I} F\right)$ in $\operatorname{Ho}(\mathcal{D})$ natural in $F: I \rightarrow \mathcal{C}$ (see 2.20). For $X \in \mathcal{C}$ and $K \in$ Spaces, define:

$$
\mathbf{K} \otimes_{l} \Phi(X) \rightarrow \Phi\left(\mathbf{K} \otimes_{l} X\right)
$$

to be the morphism hocolim $\mathbf{K} c \Phi(X) \rightarrow \Phi\left(\operatorname{hocolim}_{\mathbf{K}} c X\right)$ in $\operatorname{Ho}(\mathcal{D})$.

Corollary 9.6 Assume that $(\mathcal{C}, W)$ and $(\mathcal{D}, V)$ admit left model approximations and $\Phi: \mathcal{C} \rightarrow \mathcal{D}$ is a functor that sends weak equivalences in $\mathcal{C}$ to weak equivalences in $\mathcal{D}$. Then the induced functor $\Phi: \operatorname{Ho}(\mathcal{C}) \rightarrow \operatorname{Ho}(\mathcal{D})$ together with the morphisms $\mathbf{K} \otimes_{l} \Phi(X) \rightarrow \Phi\left(\mathbf{K} \otimes_{l} X\right)$ is a simplicial functor (2.16).

We prove finally the classical property relating mapping spaces out of a homotopy colimit with the corresponding homotopy limit of mapping spaces, see Bousfield-Kan [1, XII Proposition 4.1] and Hirschhorn [11, Theorem 19.4.4].

Proposition 9.7 Assume that $(\mathcal{C}, W)$ admits a left model approximation. Consider an object $Y$ in $\mathcal{C}$ and $F: I \rightarrow \mathcal{C}$ a functor. Then there is an isomorphism $\operatorname{map}\left(\operatorname{hocolim}_{I} F, Y\right) \cong \operatorname{holim}_{I^{\text {op }}} \operatorname{map}(F, Z)$ in Ho(Spaces) natural in $Y$ and $F$.

Proof Let us choose a left model approximation $l: \mathcal{M} \rightleftarrows \mathcal{C}: r$ and a contractible space $A$. Apply then Proposition 8.5 to a fibrant replacement $Z \in \mathcal{M}$ of $r Y$ and a cofibrant replacement $H \in \operatorname{Fun}^{b}\left(\mathbf{N}(I), \operatorname{Fun}^{b}(\mathbf{N}(\mathbf{A}), \mathcal{M})\right)$ of the bounded diagram $\mathbf{N}(I) \stackrel{\epsilon}{\rightarrow} I \stackrel{F}{\rightarrow} \mathcal{C} \stackrel{r}{\rightarrow} \mathcal{M} \stackrel{c}{\rightarrow}$ Fun $^{b}(\mathbf{N}(\mathbf{A}), \mathcal{M})$. 


\section{Right model approximations and duality}

In this section we study categories admitting both a left and a right model approximations. These structures lead to homotopy left and right actions. How related are these actions? We prove below in Theorem 10.7 that the corresponding simplicial structures on $\operatorname{Ho}(\mathcal{C})$ are isomorphic (and in particular the mapping spaces induced by the homotopy left and right actions are isomorphic). We need first some dual statements to those we have proven so far for left model approximations. They can be restated by simply taking the opposite categories.

10.1 One can define a simplicial structure on $\operatorname{Fun}^{b}\left(\mathbf{N}(\mathbf{A})^{\mathrm{op}}, \mathcal{C}\right)$ by identifying it with $\operatorname{Fun}^{b}\left(\mathbf{N}(\mathbf{A}), \mathcal{C}^{\mathrm{op}}\right)$ and applying Proposition 5.3. Explicitly, this simplicial structure is given by a functor:

$$
\text { hom: } \operatorname{Spaces}^{\mathrm{op}} \times \operatorname{Fun}^{b}\left(\mathbf{N}(\mathbf{A})^{\mathrm{op}}, \mathcal{C}\right) \rightarrow \operatorname{Fun}^{b}\left(\mathbf{N}(\mathbf{A})^{\mathrm{op}}, \mathcal{C}\right)
$$

described as follows. Denote by $\pi_{k}: \operatorname{Fun}^{b}\left(\mathbf{N}(\mathbf{K} \otimes \mathbf{A})^{\mathrm{op}}, \mathcal{C}\right) \rightarrow \operatorname{Fun}^{b}\left(\mathbf{N}(\mathbf{A})^{\mathrm{op}}, \mathcal{C}\right)$ the right adjoint of $\pi^{*}: \operatorname{Fun}^{b}\left(\mathbf{N}(\mathbf{A})^{\text {op }}, \mathcal{C}\right) \rightarrow \operatorname{Fun}^{b}\left(\mathbf{N}(\mathbf{K} \otimes \mathbf{A})^{\mathrm{op}}, \mathcal{C}\right)$, where as before $\pi: \mathbf{N}(\mathbf{K} \otimes \mathbf{A}) \rightarrow \mathbf{N}(\mathbf{A})$ stands for the subdivision of the projection onto the second factor. Define then $\operatorname{hom}(K, F):=\pi_{k} \pi^{*} F$.

10.2 Assume that $(\mathcal{C}, W)$ admits a right model approximation. Then it can be right localized. For any small category $I, \operatorname{holim}_{I}: \operatorname{Ho}(\operatorname{Fun}(I, \mathcal{C})) \rightarrow \operatorname{Ho}(\mathcal{C})$ is right adjoint to $c: \operatorname{Ho}(\mathcal{C}) \rightarrow \operatorname{Ho}(\operatorname{Fun}(I, \mathcal{C}))$. The assignment $(I, X) \mapsto \operatorname{holim}_{I} c X$ defines a functor rhom: $\mathrm{Cat}^{\mathrm{op}} \times \mathcal{C} \rightarrow \operatorname{Ho}(\mathcal{C})$. This functor is homotopy invariant: if $f: I \rightarrow J$ is a weak equivalence of small categories, then $\operatorname{rhom}(f, X)$ is an isomorphism in $\operatorname{Ho}(\mathcal{C})$ for any $X \in \mathcal{C}$ (compare with Proposition 7.1).

10.3 If $f: K \rightarrow L$ is a weak equivalence of spaces, then the induced morphism $\operatorname{rhom}(\mathbf{f}, X): \operatorname{rhom}(\mathbf{L}, X) \rightarrow \operatorname{rhom}(\mathbf{K}, X)$ is an isomorphism in $\operatorname{Ho}(\mathcal{C})$, for any $X \in \mathcal{C}$. The functor rhom: Spaces ${ }^{\text {op }} \times \mathcal{C} \rightarrow \operatorname{Ho}(\mathcal{C})$ is therefore a composition of the localization Spaces $^{\mathrm{op}} \times \mathcal{C} \rightarrow \mathrm{Ho}\left(\right.$ Spaces ${ }^{\mathrm{op}} \times \mathrm{Ho}(\mathcal{C})$ and a functor denoted by the same symbol rhom: $\operatorname{Ho}(\text { Spaces) })^{\text {op }} \times \operatorname{Ho}(\mathcal{C}) \rightarrow \operatorname{Ho}(\mathcal{C})$. This functor is a homotopy right action 2.16, ie, it has the following properties, dual to those in Corollary 7.3 and Proposition 9.4:

(1d) there are isomorphisms $X \rightarrow \operatorname{rhom}([0], X)$ in $\operatorname{Ho}(\mathcal{C})$ natural in $X$;

(2d) there are coherent isomorphisms $\operatorname{rhom}(\mathbf{K} \otimes \mathbf{L}, X) \cong \operatorname{rhom}(\mathbf{K}, \operatorname{rhom}(\mathbf{L}, X))$ in $\operatorname{Ho}(\mathcal{C})$ natural in $X \in \operatorname{Ho}(\mathcal{C})$ and $K, L \in \operatorname{Ho}$ (Spaces) ${ }^{\text {op }}$;

(3d) for any $X \in \operatorname{Ho}(\mathcal{C})$, $\operatorname{rhom}(-, X)$ : $\operatorname{Ho}(\text { Spaces) })^{\text {op }} \rightarrow \operatorname{Ho}(\mathcal{C})$ has a left adjoint $\operatorname{map}(-, X): \operatorname{Ho}(\mathcal{C}) \rightarrow \mathrm{Ho}(\text { Spaces })^{\text {op }}$. 
10.4 Let $(\mathcal{C}, W)$ and $(\mathcal{D}, V)$ be categories that admit right model approximations and $\Phi: \mathcal{C} \rightarrow \mathcal{D}$ be a functor that sends weak equivalences in $\mathcal{C}$ to weak equivalences in $\mathcal{D}$. This and the universal property of holim, as a total right derived functor, implies that, for any $F: I \rightarrow \mathcal{C}$, there is a morphism $\Phi\left(\operatorname{holim}_{I} F\right) \rightarrow \operatorname{holim}_{I} \Phi F$ in $\operatorname{Ho}(\mathcal{D})$ natural in $F$ (see 2.20). For $X \in \mathcal{C}$ and $K \in \operatorname{Spaces}$, define $\Phi(\operatorname{rhom}(\mathbf{K}, X)) \rightarrow \operatorname{rhom}(\mathbf{K}, \Phi F)$ to be the morphism $\Phi\left(\operatorname{holim}_{\mathbf{K}} c X\right) \rightarrow \operatorname{holim}_{\mathbf{K}} c \Phi X$ in $\operatorname{Ho}(\mathcal{D})$. In this way the functor $\Phi: \operatorname{Ho}(\mathcal{C}) \rightarrow \operatorname{Ho}(\mathcal{D})$ together with the morphism $\Phi(\operatorname{rhom}(\mathbf{K}, X)) \rightarrow \operatorname{rhom}(\mathbf{K}, \Phi F)$ is a simplicial functor.

10.5 The functor $\operatorname{map}(-, X): \operatorname{Ho}(\mathcal{C}) \rightarrow \mathrm{Ho}(\text { Spaces })^{\text {op }}$, defined in 10.3 , can be rigidified as follows:

- choose a right model approximation $l: \mathcal{C} \rightleftarrows \mathcal{M}: r$;

- choose a contractible space $A$;

- choose a fibrant replacement $R$ in $\operatorname{Fun}^{b}\left(\mathbf{N}(\mathbf{A})^{\mathrm{op}}, \mathcal{M}\right)$ and a cofibrant replacement $Q$ in $\mathcal{M}$;

- define a functor map: $\mathcal{C}^{\mathrm{op}} \times \mathcal{C} \rightarrow$ Spaces by the formula:

$$
\operatorname{map}(X, Y):=\operatorname{map}(c Q l(X), \operatorname{Rcr}(Y))
$$

where the mapping space on the right comes from the simplicial structure on $\operatorname{Fun}^{b}\left(\mathbf{N}(\mathbf{A})^{\mathrm{op}}, \mathcal{M}\right)$.

The functor map: $\mathcal{C}^{\mathrm{op}} \times \mathcal{C} \rightarrow$ Spaces, defined by the above procedure, is homotopy invariant and its values are Kan spaces. The induced functor on the level of homotopy categories is naturally isomorphic to the functor described in 10.3 and therefore it does not depend on the choice of the right model approximation $l: \mathcal{C} \rightleftarrows \mathcal{M}: r$, the contractible space $A$, and the fibrant and cofibrant replacements.

10.6 The mapping space into a homotopy limit satisfies a property analogous to Proposition 9.7. Let $Y \in \mathcal{C}$ and $F: I \rightarrow \mathcal{C}$ be a functor. Then there is an isomorphism $\operatorname{map}\left(Y, \operatorname{holim}_{I} F\right) \cong \operatorname{holim}_{I} \operatorname{map}(Z, F)$ in Ho(Spaces) natural in $Y$ and $F$.

We are now ready to compare the homotopy left and right actions arising from left and right model approximations.

Theorem 10.7 Assume that $(\mathcal{C}, W)$ admits both a left and a right model approximations. Then, for any space $K$, the functor $\mathbf{K} \otimes_{l}-: \operatorname{Ho}(\mathcal{C}) \rightarrow \operatorname{Ho}(\mathcal{C})$ is left adjoint to $\operatorname{rhom}(\mathbf{K},-): \operatorname{Ho}(\mathcal{C}) \rightarrow \operatorname{Ho}(\mathcal{C})$. The weak simplicial structures on $\operatorname{Ho}(\mathcal{C}) 2.15$, induced by the homotopy actions $\otimes_{l}$ and rhom, are isomorphic. In particular the induced mapping spaces are also isomorphic. 
Proof According to Corollary 6.7, we have the following adjoint pairs:

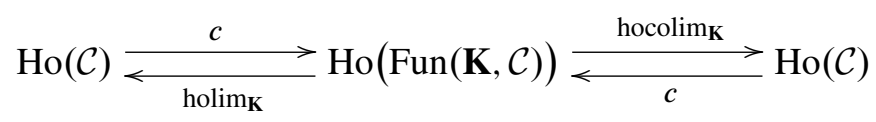

Since, by definition, $\operatorname{hocolim}_{\mathbf{K}} c X=\mathbf{K} \otimes_{l} X$ and $\operatorname{holim}_{\mathbf{K}} c X=\operatorname{rhom}(\mathbf{K}, X)$ the theorem is proven.

\section{Monoidal model categories}

Recall that a monoidal model category is a model category $\mathcal{M}$ together with a "compatible" monoidal structure $\mathcal{M} \otimes \mathcal{M} \rightarrow \mathcal{M}$, see [12, Definition 4.2.6]. Hovey asks whether the homotopy category of such a monoidal model category always forms a central algebra over Ho(Spaces), ie if there is a monoidal functor $i$ : Ho(Spaces) $\rightarrow \operatorname{Ho}(\mathcal{M})$ and natural isomorphisms $t: i(K) \otimes X \rightarrow X \otimes i(K)$ satisfying certain coherence conditions introduced in [12, Definition 4.1.10]. We show this is indeed so.

Proposition 11.1 Let $\mathcal{M}$ be a monoidal model category. Then the functor

$$
\otimes_{l}: \operatorname{Ho}(\text { Spaces }) \times \operatorname{Ho}(\mathcal{M}) \rightarrow \operatorname{Ho}(\mathcal{M})
$$

describes a central algebra over Ho(Spaces).

Proof Let us denote by $\otimes_{\mathcal{M}}: \mathcal{M} \times \mathcal{M} \rightarrow \mathcal{M}$ the monoidal structure in $\mathcal{M}$ and by $S \in \mathcal{M}$ the unit. By definition, for any cofibrant $X \in \mathcal{M}$, the operations $X \otimes_{\mathcal{M}}-$ and $-\otimes_{\mathcal{M}} X$ preserve cofibrations. Consequently, for any such $X$, if $F \in \operatorname{Fun}^{b}(A, \mathcal{M})$ is cofibrant, then so are the functors $X \otimes_{\mathcal{M}} F$ and $F \otimes_{\mathcal{M}} X$.

Let $F: I \rightarrow \mathcal{M}$ be a functor and $Q F \in \operatorname{Fun}^{b}(\mathbf{N}(I), \mathcal{M})$ be a cofibrant replacement of the composition of $\epsilon: \mathbf{N}(I) \rightarrow I$ and $F$. The above remark implies that, for a cofibrant $X, Q F \otimes_{\mathcal{M}} X$ and $X \otimes_{\mathcal{M}} Q F$ are cofibrant replacements of $F \epsilon \otimes_{\mathcal{M}} X$ and $X \otimes_{\mathcal{M}} F \epsilon$ respectively. Since $\otimes_{\mathcal{M}}$ commutes with colimits we have isomorphisms in $\mathcal{M}:$

$$
\begin{aligned}
& \operatorname{colim}_{\mathrm{N}(I)}\left(Q F \otimes_{\mathcal{M}} X\right) \cong\left(\operatorname{colim}_{\mathrm{N}(I)} Q F\right) \otimes_{\mathcal{M}} X \\
& \operatorname{colim}_{\mathrm{N}(I)}\left(X \otimes_{\mathcal{M}} Q F\right) \cong X \otimes_{\mathcal{M}}\left(\operatorname{colim}_{\mathrm{N}(I)} Q F\right) .
\end{aligned}
$$

The corresponding isomorphisms in $\operatorname{Ho}(\mathcal{M})$ are easily seen to be natural in $F: I \rightarrow \mathcal{M}$ and $X \in \operatorname{Ho}(\mathcal{M})$ :

$$
\begin{aligned}
& \operatorname{hocolim}_{I}\left(F \otimes_{\mathcal{M}} X\right) \cong\left(\operatorname{hocolim}_{I} F\right) \otimes_{\mathcal{M}} X \\
& \operatorname{hocolim}_{I}\left(X \otimes_{\mathcal{M}} F\right) \cong X \otimes_{\mathcal{M}}\left(\operatorname{hocolim}_{I} F\right) .
\end{aligned}
$$

Algebraic $8 \mathcal{G}$ Geometric Topology, Volume 8 (2008) 
It is now straightforward to check that the following isomorphism $t$ :

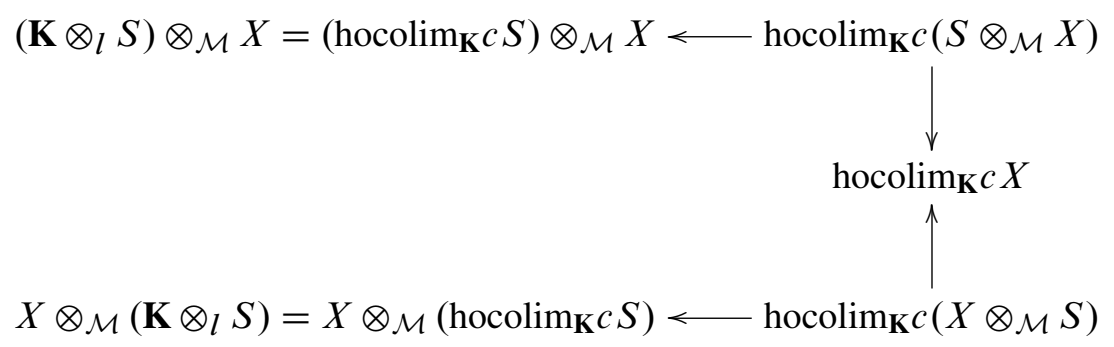

satisfies the coherence conditions for $\operatorname{Ho}(\mathcal{M})$ to be central, where the monoidal functor $i$ is defined by $i(K)=\mathbf{K} \otimes_{l} S$.

\section{References}

[1] A K Bousfield, D M Kan, Homotopy limits, completions and localizations, Lecture Notes in Mathematics 304, Springer, Berlin (1972) MR0365573

[2] W Chachólski, J Scherer, Homotopy theory of diagrams, Mem. Amer. Math. Soc. 155 (2002) MR1879153

[3] D-C Cisinski, Images directes cohomologiques dans les catégories de modèles, Ann. Math. Blaise Pascal 10 (2003) 195-244 MR2031269

[4] D-C Cisinski, Propriétés universelles et extensions de Kan dérivées (2003) Preprint

[5] D Dugger, Replacing model categories with simplicial ones, Trans. Amer. Math. Soc. 353 (2001) 5003-5027 MR1852091

[6] W G Dwyer, PS Hirschhorn, D M Kan, J H Smith, Homotopy limit functors on model categories and homotopical categories, Mathematical Surveys and Monographs 113, American Mathematical Society, Providence, RI (2004) MR2102294

[7] W G Dwyer, D M Kan, Function complexes in homotopical algebra, Topology 19 (1980) 427-440 MR584566

[8] W G Dwyer, J Spaliński, Homotopy theories and model categories, from: "Handbook of algebraic topology", North-Holland, Amsterdam (1995) 73-126 MR1361887

[9] T G Goodwillie, Calculus. II. Analytic functors, K-Theory 5 (1991/92) 295-332 MR1162445

[10] A Heller, Homotopy theories, Mem. Amer. Math. Soc. 71 (1988) MR920963

[11] PS Hirschhorn, Model categories and their localizations, Mathematical Surveys and Monographs 99, American Mathematical Society, Providence, RI (2003) MR1944041

[12] M Hovey, Model categories, Mathematical Surveys and Monographs 63, American Mathematical Society, Providence, RI (1999) MR1650134 
[13] D M Kan, On c. s. s. complexes, Amer. J. Math. 79 (1957) 449-476 MR0090047

[14] D M Latch, R W Thomason, W S Wilson, Simplicial sets from categories, Math. Z. 164 (1979) 195-214 MR516607

[15] S Mac Lane, Categories for the working mathematician, second edition, Graduate Texts in Mathematics 5, Springer, New York (1998) MR1712872

[16] D G Quillen, Homotopical algebra, Lecture Notes in Mathematics 43, Springer, Berlin (1967) MR0223432

[17] C Rezk, S Schwede, B Shipley, Simplicial structures on model categories and functors, Amer. J. Math. 123 (2001) 551-575 MR1833153

K.T.H., Matematik, S-10044 Stockholm, Sweden

Universitat Autònoma de Barcelona, Departament de Matemàtiques,

E-08193 Bellaterra, Spain

wojtek@math.kth.se, jscherer@mat.uab.cat

Received: 5 September 2007 Revised: 19 December 2007 